\title{
Synthesis, Characterisation and In Vitro Biological Evaluation of Novel 3-Chloro-1-(5-ethyl-[1,3,4] thiadiazole-2-yl)-4-phenyl-azetidin-2-one Derivatives
}

\author{
SUNIL MAKWANE ${ }^{1 *}$, S. D. SRIVASTAVA ${ }^{1}$, RAJIV DUA ${ }^{2}$ and S. K. SRIVASTAVA ${ }^{1}$
}

${ }^{1}$ Synthetic Organic Chemistry Laboratory, Department of Chemistry, School of Chemical Sciences and Technology, Dr. Harisingh Gour University (A Central University), Sagar (M.P), India

${ }^{2}$ Department of Chemistry, State Forensic Science Laboratory, Department of Home (Police), Government of Madhya Pradesh, Sagar (M.P), India

sanumkwn@gmail.com

Received 3 May 2018 / Accepted 25 May 2018

\begin{abstract}
The 1,3,4-thiadiazole molecules are interesting significance in the field of health pharmaceutics and agriculture. Derivatives of 2-amino-5-ethyl-1,3,4-thiadiazole obtained by cyclization from chloroacetyl chloride in the presence of triethyl amine. Schiff base of 2-amino-5ethyl-1,3,4-thiadiazole on reaction with aromatic aldehydes afforded compound (1). This compound on further reaction with chloroacetyl chloride in the presence of $\mathrm{Et}_{3} \mathrm{~N}$ yielded 3-chloro-1-(5-ethyl$[1,3,4]$ thiadiazole-2-yl)-4methyl-azetidin-2-ones (2). Antibacterial and antifungal activities were performed on Bacillus subtilis, Escherichia coli and $S$ tyhpi bacteria and Aspergillus niger, Aspergillus flavus and Fusarium oxisporium fungi respectively. Structures of all the synthesized compounds were confirmed using IR, ${ }^{1} \mathrm{H}$ NMR and ${ }^{13} \mathrm{C}$ NMR and MS spectroscopy.
\end{abstract}

Keywords: Thiadiazole, Azetidine, Antimicrobial and Antifungal

\section{Introduction}

Heterocyclic chemistry offers an example for the lack of distinct demarcations; in fact, it pervades the plurality of the other chemical disciplines. Heterocycles are inextricably woven into the life processes. The vital interest of the pharmaceutical and agrochemical industries in heterocycles is often connected with their natural occurrence ${ }^{1}$. A survey of the literature revealed that heterocyclic compound differently substituted 1,3,4-thiadiazoles and annulated 1,3,4-thiadiazoles have wide range of pharmacological activities such as antibacterial, antifungal $^{2}$, antituberculosis ${ }^{3}, \quad$ antileishmanial $^{4}, \quad$ anti-inflammatory $^{5}$, analgesic $^{6}$, antidepressant $^{7}$, antitumor ${ }^{8}$, antioxidant ${ }^{9}$ and anticonvulsant activities ${ }^{10}$. The 2-azetidinone ring system, a common structural feature of a number of wide spectrum $\beta$-lactam antibiotics, including penicillins, cephalosporins, carbapenems, nocardicins and monobactams, which 
have been widely used as chemotherapeutic agents to treat bacterial infections and microbial diseases ${ }^{11}$. Recently 2 -azetidinone has been assigned for good antimicrobial ${ }^{12}$, antitubercular ${ }^{13}$, anti-inflammatory, anticonvulsant ${ }^{14}$, cardiovascular $^{15}$ and antioxidant activities ${ }^{16}$. By the considering the above argument we have synthesized several novel 1,3,4-thiadiazoleazetidin-2-ones compounds in order to study their expellant biological behavior. The present study report that synthesis of benzylidene-(5-ethyl-[1,3,4]thiadiazol-2-yl)-amine $\mathbf{1}$ and $\mathbf{1 a - 1} \boldsymbol{l}$ and 3-chloro-1-(5-ethyl-[1,3,4]thiadiazol-2-yl)-4-phenyl-azetidin-2-one, $\mathbf{2}$ and $\mathbf{2 a - 2 l}$ by the appropriate synthetic methods (Scheme 1). All the final product of the synthesized compounds have been screened for their in vitro antifungal activity against $A$ niger, $P$. Citrinum, $F$ oxysporium and anti-microbial activity against E. coli, B. Subtilis and S. Typhi respectively ${ }^{17,18}$.
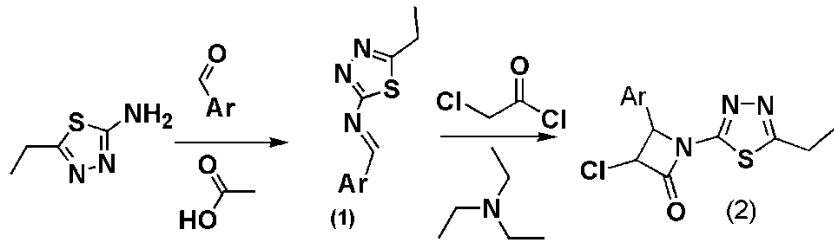

$\mathrm{Ar}=$ various substituted aryl groups

Scheme 1. Synthesis of compounds $(1 \&$ 2)

\section{Experimental}

All the chemicals and reagents were of analytical grade of sigma Aldrich, Merck, Chemi-loba and Himedia. The reagents and solvents were purified before using by standard methods. Melting points were taken in open capillaries and are uncorrected. Progress of reaction was monitored at various stages by silica gel-G coated TLC plates using $\mathrm{MeOH}$ : $\mathrm{CHCl}_{3}$ system. The spot was visualized by exposing dry plate at iodine vapour chamber and fluorescent indicator F 254 UV chamber. IR spectra were recorded in KBr disc on a Schimadzu8201 PC, FTIR spectrophotometer $\left(v\right.$ max in $\left.\mathrm{cm}^{-1}\right)$ and ${ }^{1} \mathrm{H}$ NMR and ${ }^{13} \mathrm{C}$ NMR spectra were measured on a Brucker DRX-300 spectrometer in $\mathrm{CDCl}_{3}$ at 500 and $75 \mathrm{MHz}$ respectively using TMS as an internal standard. All chemical shifts were reported on $\delta$ scale. The mass spectra were recorded on a Jeol SX-102 GC-MS mass spectrometer. Elemental analysis was performed on a Carlo Erba-1108 analyzer. The analytical data of all the compounds were highly satisfactory (Table 1). All the synthesized compounds were purified by column chromatography using Merck silica Gel 60 (230-400 Mesh). The reagent grade chemicals were purchased from the commercial sources.

\section{Synthesis of the benzylidene-(5-ethyl-[1,3,4]thiadiazol-2-yl)amine (1)}

Benzylidene-(5-ethyl-[1,3,4]thiadiazol-2-yl)amine was synthesized (Figure 1) by using equimolar reaction of 2-amino-5-ethyl-1,3,4-thiadiazole (0.004 mole) and benzaldehyde ( 0.004 mole) in toluene $(25 \mathrm{~mL})$, followed by continuous stirring for about $1 \mathrm{~h}$ at room temperature. Then reaction mixture was refluxed on a heating mantle in Dean-Stark apparatus for about $2 \mathrm{~h}$ using glacial acetic acid as a catalyst. In the reaction mixture molecular sieves were used to trap for produced water molecule. After the completion of the reaction, the flask was removed from Dean-Stark apparatus and excess of solvent was recovered by simple distillation method under reduced pressure using heating mental at about $115-120{ }^{\circ} \mathrm{C}$. A solid product was obtained which was purified over a silica gel column using chloroform: methanol $(8: 2 \mathrm{v} / \mathrm{v})$ mixture as eluant. The elute was concentrated to give a product which was recrystallized from ethanol at room temperature to yield compound 1. White crystalline solid, M.P. $190-192{ }^{\circ} \mathrm{C}$, Yield 75\%, IR: $\left(v_{\max } \mathrm{cm}^{-1}\right)$ 1453( $\left.v_{\mathrm{C}-\mathrm{C}}\right), 712\left(v_{\mathrm{C}-\mathrm{S}}\right), 1642\left(v_{\mathrm{N}=\mathrm{C}}\right), 1430\left(v_{\mathrm{C}-\mathrm{N}}\right), 3118\left(v_{\mathrm{C}-\mathrm{H}}\right), 1310\left(v_{\mathrm{N}-\mathrm{N}}\right)$. 
${ }^{1} \mathrm{H}$ NMR: $\delta(\mathrm{ppm}) 1.20\left(3 \mathrm{H}, \mathrm{CH}_{3} \mathrm{t}, \mathrm{J}=7.3 \mathrm{~Hz}\right), 3.06\left(2 \mathrm{H}, \mathrm{CH}_{2}, \mathrm{~N}=\mathrm{CHq}, \mathrm{J}=7.3 \mathrm{~Hz}\right), 7.40-8.06$ $(5 \mathrm{H}, \mathrm{Ar}-\mathrm{H}, \mathrm{m}),, 9.39(1 \mathrm{H}, \mathrm{s}, \mathrm{N}=\mathrm{CH}$ acyclic $),{ }^{13} \mathrm{C}$ NMR: $\delta(\mathrm{ppm}) 12.35\left(\mathrm{CH}_{3}\right.$ acyclic $), 27.93$ $\left(\mathrm{CH}_{2}\right.$ acyclic),127.7-134.61(6C of aromatic ring), 158.91( $\mathrm{N}=\mathrm{CH}$ acyclic), $159.6(\mathrm{CH}$ acyclic, $) 160.4,156.7\left(\mathrm{C}_{2}, \mathrm{C}_{5}\right.$ of thiadiazole), Anal. Calcd. For: $\mathrm{C}_{11} \mathrm{H}_{11} \mathrm{~N}_{3} \mathrm{~S}: \mathrm{C}, 60.80, \mathrm{H}, 5.10$, $\mathrm{N}, 19.34 \%$, found C, 60.76, H,5.77, N,19.4\% ; Mass $217.07\left(\mathrm{M}^{+}\right)$.

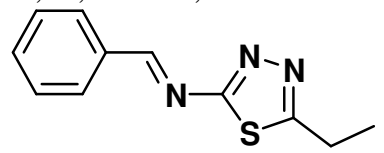

Figure 1. Structure of compound $\mathbf{1}$

The compounds $\mathbf{1 a - 1} \mathbf{l}$ were synthesized by the similar method as reported earlier.

Table 1. Characterization data of the compound $\mathbf{1}$ and $\mathbf{1 a - 1} \boldsymbol{l}$ and $\mathbf{2}$ and $\mathbf{2 a}-\mathbf{2 l}$

\begin{tabular}{|c|c|c|c|c|c|}
\hline Compd. & Ar1 & Yield, \% & M.P. ${ }^{0} \mathrm{C}$ & $\begin{array}{l}\text { Molecular } \\
\text { Formulae }\end{array}$ & $\begin{array}{l}\text { Mass spectra } \\
\left(\mathrm{M}^{+}\right)\end{array}$ \\
\hline 1 & $\mathrm{C}_{6} \mathrm{H}_{5}$ & 75 & $190-92$ & $\mathrm{C}_{11} \mathrm{H}_{11} \mathrm{~N}_{3} \mathrm{~S}$ & 217.07 \\
\hline $1 \mathbf{a}$ & $2-\mathrm{ClC}_{6} \mathrm{H}_{4}$ & 66 & $175-77$ & $\mathrm{C}_{11} \mathrm{H}_{10} \mathrm{ClN}_{3} \mathrm{~S}$ & 251.03 \\
\hline $1 b$ & $3-\mathrm{ClC}_{6} \mathrm{H}_{4}$ & 62 & $172-75$ & $\mathrm{C}_{11} \mathrm{H}_{10} \mathrm{ClN}_{3} \mathrm{~S}$ & 251.03 \\
\hline 1c & $4-\mathrm{ClC}_{6} \mathrm{H}_{4}$ & 67 & $173-76$ & $\mathrm{C}_{11} \mathrm{H}_{10} \mathrm{ClN}_{3} \mathrm{~S}$ & 251.04 \\
\hline 1d & $2-\mathrm{BrC}_{6} \mathrm{H}_{4}$ & 71 & $180-81$ & $\mathrm{C}_{11} \mathrm{H}_{10} \mathrm{BrN}_{3} \mathrm{~S}$ & 294.95 \\
\hline 1e & $3-\mathrm{BrC}_{6} \mathrm{H}_{4}$ & 72 & $181-82$ & $\mathrm{C}_{11} \mathrm{H}_{10} \mathrm{BrN}_{3} \mathrm{~S}$ & 294.96 \\
\hline 1f & $4-\mathrm{BrC}_{6} \mathrm{H}_{4}$ & 68 & $182-83$ & $\mathrm{C}_{11} \mathrm{H}_{10} \mathrm{BrN}_{3} \mathrm{~S}$ & 294.98 \\
\hline $1 g$ & $2-\mathrm{NO}_{2} \mathrm{C}_{6} \mathrm{H}_{4}$ & 80 & $176-79$ & $\mathrm{C}_{11} \mathrm{H}_{10} \mathrm{~N}_{4} \mathrm{O}_{2} \mathrm{~S}$ & 273.06 \\
\hline $1 \mathrm{~h}$ & $3-\mathrm{NO}_{2} \mathrm{C}_{6} \mathrm{H}_{4}$ & 79 & $175-77$ & $\mathrm{C}_{11} \mathrm{H}_{10} \mathrm{~N}_{4} \mathrm{O}_{2} \mathrm{~S}$ & 273.04 \\
\hline $\mathbf{1 i}$ & $4-\mathrm{NO}_{2} \mathrm{C}_{6} \mathrm{H}_{4}$ & 81 & $176-78$ & $\mathrm{C}_{11} \mathrm{H}_{10} \mathrm{~N}_{4} \mathrm{O}_{2} \mathrm{~S}$ & 275.02 \\
\hline $\mathbf{1 j}$ & $2-\mathrm{OCH}_{3} \mathrm{C}_{6} \mathrm{H}_{4}$ & 61 & $136-37$ & $\mathrm{C}_{12} \mathrm{H}_{13} \mathrm{~N}_{3} \mathrm{OS}$ & 258.07 \\
\hline $1 \mathbf{k}$ & $3-\mathrm{OCH}_{3} \mathrm{C}_{6} \mathrm{H}_{4}$ & 59 & $134-36$ & $\mathrm{C}_{12} \mathrm{H}_{13} \mathrm{~N}_{3} \mathrm{OS}$ & 258.05 \\
\hline $1 l$ & $4-\mathrm{OCH}_{3} \mathrm{C}_{6} \mathrm{H}_{4}$ & 57 & $136-38$ & $\mathrm{C}_{12} \mathrm{H}_{13} \mathrm{~N}_{3} \mathrm{OS}$ & 258.03 \\
\hline 2 & $\mathrm{C}_{6} \mathrm{H}_{5}$ & 72 & $189-191$ & $\mathrm{C}_{13} \mathrm{H}_{12} \mathrm{ClN}_{3} \mathrm{OS}$ & 293.03 \\
\hline $2 a$ & $2-\mathrm{ClC}_{6} \mathrm{H}_{4}$ & 72 & $189-191$ & $\mathrm{C}_{13} \mathrm{H}_{11} \mathrm{Cl}_{2} \mathrm{~N}_{3} \mathrm{OS}$ & 327.00 \\
\hline $2 b$ & $3-\mathrm{ClC}_{6} \mathrm{H}_{4}$ & 66 & $172-175$ & $\mathrm{C}_{13} \mathrm{H}_{11} \mathrm{Cl}_{2} \mathrm{~N}_{3} \mathrm{OS}$ & 327.01 \\
\hline $2 c$ & 4- $\mathrm{ClC}_{6} \mathrm{H}_{4}$ & 67 & $173-175$ & $\mathrm{C}_{13} \mathrm{H}_{11} \mathrm{Cl}_{2} \mathrm{~N}_{3} \mathrm{OS}$ & 327.00 \\
\hline 2d & $2-\mathrm{BrC}_{6} \mathrm{H}_{4}$ & 71 & $178-180$ & $\mathrm{C}_{13} \mathrm{H}_{11} \mathrm{Br}_{2} \mathrm{~N}_{3} \mathrm{OS}$ & 370.95 \\
\hline $2 e$ & $3-\mathrm{BrC}_{6} \mathrm{H}_{4}$ & 75 & $180-182$ & $\mathrm{C}_{13} \mathrm{H}_{11} \mathrm{BrClN}_{3} \mathrm{OS}$ & 370.92 \\
\hline $2 f$ & $4-\mathrm{BrC}_{6} \mathrm{H}_{4}$ & 68 & $180-182$ & $\mathrm{C}_{13} \mathrm{H}_{11} \mathrm{BrClN}_{3} \mathrm{OS}$ & 370.92 \\
\hline $2 g$ & $2-\mathrm{NO}_{2} \mathrm{C}_{6} \mathrm{H}_{4}$ & 80 & $176-179$ & $\mathrm{C}_{13} \mathrm{H}_{11} \mathrm{ClN}_{4} \mathrm{O}_{3} \mathrm{~S}$ & 338.02 \\
\hline $2 \mathrm{~h}$ & $3-\mathrm{NO}_{2} \mathrm{C}_{6} \mathrm{H}_{4}$ & 79 & $175-177$ & $\mathrm{C}_{13} \mathrm{H}_{11} \mathrm{ClN}_{4} \mathrm{O}_{3} \mathrm{~S}$ & 338.03 \\
\hline $2 \mathbf{i}$ & $4-\mathrm{NO}_{2} \mathrm{C}_{6} \mathrm{H}_{4}$ & 81 & $183-185$ & $\mathrm{C}_{13} \mathrm{H}_{11} \mathrm{ClN}_{4} \mathrm{O}_{3} \mathrm{~S}$ & 338.01 \\
\hline $2 \mathbf{j}$ & $2-\mathrm{OCH}_{3} \mathrm{C}_{6} \mathrm{H}_{4}$ & 64 & $139-141$ & $\mathrm{C}_{14} \mathrm{H}_{14} \mathrm{ClN}_{3} \mathrm{O}_{2} \mathrm{~S}$ & 323.05 \\
\hline $2 \mathbf{k}$ & $3-\mathrm{OCH}_{3} \mathrm{C}_{6} \mathrm{H}_{4}$ & 67 & $140-142$ & $\mathrm{C}_{14} \mathrm{H}_{14} \mathrm{ClN}_{3} \mathrm{O}_{2} \mathrm{~S}$ & 323.04 \\
\hline $2 l$ & $4-\mathrm{OCH}_{3} \mathrm{C}_{6} \mathrm{H}_{4}$ & 65 & $138-140$ & $\mathrm{C}_{14} \mathrm{H}_{14} \mathrm{ClN}_{3} \mathrm{O}_{2} \mathrm{~S}$ & 323.06 \\
\hline
\end{tabular}

2-Chloro-benzylidene)-(5-ethyl-[1,3,4]thiadiazol-2-yl)-amine (1a)

M.P. $175-177{ }^{0} \mathrm{C}$, Yield $66 \%, \operatorname{IR}\left(v_{\max } \mathrm{cm}^{-1}\right) 1453\left(v_{\mathrm{C}-\mathrm{C}}\right), 742\left(v_{\mathrm{C}-\mathrm{S}}\right), 1647\left(v_{\mathrm{N}}=\mathrm{C}\right), 1430\left(v_{\mathrm{C}-\mathrm{N}}\right)$, 2989 $\left(v_{\mathrm{C}-\mathrm{H}}\right), 1310\left(v_{\mathrm{N}-\mathrm{N}}\right),{ }^{1} \mathrm{H}$ NMR: $\delta(\mathrm{ppm}) 1.22\left(3 \mathrm{H}, \mathrm{t}, \mathrm{J}=7.3 \mathrm{~Hz}, \mathrm{CH}_{3}\right), 3.03(2 \mathrm{H}, \mathrm{q}, \mathrm{J}=$ $7.3 \mathrm{~Hz} \mathrm{CH})_{2}, 7.35-8.05(4 \mathrm{H}, \mathrm{m}) ,9.41(1 \mathrm{H}, \mathrm{s}, \mathrm{N}=\mathrm{CH}),{ }^{13} \mathrm{C} \mathrm{NMR} \delta(\mathrm{ppm}) 13.05\left(\mathrm{CH}_{3}\right.$ acyclic $)$, $27.93\left(\mathrm{CH}_{2}\right.$ acyclic), 128.8-136.7 (6C of aromatic ring), 136.7, 159.50 ( $\mathrm{N}=\mathrm{CH}$ acyclic), 156.7, 160.4,156.7 $\left(\mathrm{C}_{2}, \mathrm{C}_{5}\right.$ of thiadiazole), Anal. Calcd. For : $\mathrm{C}_{11} \mathrm{H}_{10} \mathrm{~N}_{3} \mathrm{~S}: \mathrm{C}, 52.48, \mathrm{H}, 4.00$, $\mathrm{N}, 16.69 \%$, found $\mathrm{C}, 52.28, \mathrm{H}, 3.97, \mathrm{~N}, 16.54 \%$, Mass $251.03\left(\mathrm{M}^{+}\right)$. 
3-Chloro-benzylidene)-(5-ethyl-[1,3,4]thiadiazol-2-yl)-amine (1b)

M.P. $172-175{ }^{0} \mathrm{C}$, Yield $62 \%$, IR : $\left(v_{\max } \mathrm{cm}^{-1}\right) 1543\left(v_{\mathrm{C}-\mathrm{C}}\right), 752\left(v_{\mathrm{C}-\mathrm{S}}\right), 1655\left(v_{\mathrm{N}=\mathrm{C}}\right), 1432\left(v_{\mathrm{C}-\mathrm{N}}\right)$, $3018\left(v_{\mathrm{C}-\mathrm{H}}\right), 1310\left(v_{\mathrm{N}-\mathrm{N}}\right), 719\left(v_{\mathrm{C}-\mathrm{Cl}}\right) .{ }^{1} \mathrm{H}$ NMR: $\delta(\mathrm{ppm}) 1.20(3 \mathrm{H}, \mathrm{t}, \mathrm{J}=7.3 \mathrm{~Hz} \mathrm{CH}), 3.04(2 \mathrm{H}$, $\mathrm{q}, \mathrm{J}=7.3 \mathrm{~Hz} \mathrm{CH}$ ), 7.46-7.99 $(3 \mathrm{H}, \mathrm{m}), 7.92(1 \mathrm{H}, \mathrm{dt}, \mathrm{J}=7.8,1.2 \mathrm{~Hz}), 9.43(1 \mathrm{H}, \mathrm{s}, \mathrm{N}=\mathrm{CH}$ acyclic), ${ }^{13} \mathrm{C}$ NMR: $\delta$ (ppm) $12.55\left(\mathrm{CH}_{3}\right.$ acyclic), $26.93\left(\mathrm{CH}_{2}\right.$ acyclic), 127.8-130.7 (C of aromatic ring), $159.61\left(\mathrm{~N}=\mathrm{CH}\right.$ acyclic), $158.4,156.7\left(\mathrm{C}_{2}, \mathrm{C}_{5}\right.$ of thiadiazole), Anal. Calcd. for: $\mathrm{C}_{11} \mathrm{H}_{10} \mathrm{Cl} \mathrm{N}{ }_{3} \mathrm{~S}: \mathrm{C}, 52.48, \mathrm{H}, 4.00,14.08, \mathrm{~N}, 16.6974 \%$, found $\mathrm{C}, 52.18, \mathrm{H}, 3.92, \mathrm{~N}, 16.50 \%$, Mass 251.03( $\left.\mathrm{M}^{+}\right)$.

\section{4-Chloro-benzylidene)-(5-ethyl-[1,3,4]thiadiazol-2-yl)-amine (1c)}

M.P.173-176 ${ }^{0} \mathrm{C}$, Yield $67 \%$, IR: $\left(v_{\max } \mathrm{cm}^{-1}\right) 1549\left(v_{\mathrm{C}-\mathrm{C}}\right), 746\left(v_{\mathrm{C}-\mathrm{S}}\right), 1661\left(v_{\mathrm{N}=\mathrm{C}}\right), 1439\left(v_{\mathrm{C}-\mathrm{N}}\right)$, $3108\left(v_{\mathrm{C}-\mathrm{H}}\right), 1316\left(v_{\mathrm{N}-\mathrm{N}}\right), 717\left(v_{\mathrm{C}-\mathrm{Cl}}\right) .{ }^{1} \mathrm{H}$ NMR: $\delta(\mathrm{ppm}) 1.26\left(3 \mathrm{H}, \mathrm{t}, \mathrm{J}=7.3 \mathrm{~Hz} \mathrm{CH}_{3}\right), 3.03(2 \mathrm{H}$, $\mathrm{q}, \mathrm{J}=7.3 \mathrm{~Hz} \mathrm{CH}$ ), 7.66-8.00 (4H, m, Ar-H), $9.37(1 \mathrm{H}, \mathrm{s}, \mathrm{N}=\mathrm{CH}),{ }^{13} \mathrm{C}$ NMR : $\delta(\mathrm{ppm})$ 12.75 $\left(\mathrm{CH}_{3}\right.$ acyclic), $27.93\left(\mathrm{CH}_{2}\right.$ acyclic, )129.42-135.7 ( $\mathrm{C}$ of aromatic ring), $159.81(\mathrm{~N}=\mathrm{CH}$ acyclic), 158.9, $156.7\left(\mathrm{C}_{2}, \mathrm{C}_{5}\right.$ of thiadiazole), Anal. Calcd. for: $\mathrm{C}_{11} \mathrm{H}_{10} \mathrm{ClN}_{3} \mathrm{~S}: \mathrm{C}, 52.48, \mathrm{H}$, 4.00, N, $16.69 \%$, found C, 52.24, H, 3.97, N, 16.51\%, Mass 251.04( $\left.\mathrm{M}^{+}\right)$.

\section{2-Bromo-benzylidene)-(5-ethyl-[1,3,4]thiadiazol-2-yl)-amine (1d)}

M.P.180-181 ${ }^{\circ}$ C, Yield $71 \%$, IR: $\left(v_{\max } \mathrm{cm}^{-1}\right)-1546\left(v_{\mathrm{C}-\mathrm{C}}\right), 741\left(v_{\mathrm{C}-\mathrm{S}}\right), 1664\left(v_{\mathrm{N}=\mathrm{C}}\right), 1440\left(v_{\mathrm{C}-\mathrm{N}}\right)$, $2950\left(v_{\mathrm{C}-\mathrm{H}}\right), 1320\left(v_{\mathrm{N}-\mathrm{N}}\right), 549\left(v_{\mathrm{C}-\mathrm{Br}}\right){ }^{1} \mathrm{H} \mathrm{NMR}: \delta(\mathrm{ppm}) 1.20\left(3 \mathrm{H}, \mathrm{t}, \mathrm{J}=6.6 \mathrm{~Hz}, \mathrm{CH}_{3}\right), 3.04(2 \mathrm{H}$, $\left.\mathrm{q}, \mathrm{J}=6.6 \mathrm{~Hz}, \mathrm{CH}_{2}\right), 7.29-7.91(4 \mathrm{H}, \mathrm{m}, \mathrm{Ar}-\mathrm{H}), 9.39(1 \mathrm{H}, \mathrm{s}, \mathrm{N}=\mathrm{CH}),{ }^{13} \mathrm{C} \mathrm{NMR}: \delta(\mathrm{ppm})$ $12.65\left(\mathrm{CH}_{3}\right.$ acyclic $), 28.83\left(\mathrm{CH}_{2}\right.$ acyclic), $159.51(\mathrm{~N}=\mathrm{CH}$ acyclic $), 127.42-132.19(\mathrm{C}$ of aromatic ring), 159.4,155.7 $\left(\mathrm{C}_{2}, \mathrm{C}_{5}\right.$ of thiadiazole), Anal. Calcd for: $\mathrm{C}_{11} \mathrm{H}_{10} \mathrm{Br} \mathrm{N} \mathrm{N}_{3} \mathrm{~S}: \mathrm{C}, 44.61$, H, 3.40, N, 14.19, \%, found C, 44.21, H, 3.27, N, $14.11 \%$, Mass $294.95\left(\mathrm{M}^{+}\right)$.

\section{3-Bromo-benzylidene)-(5-ethyl-[1,3,4]thiadiazol-2-yl)-amine (1e)}

M.P. $180-182^{0} \mathrm{C}$, Yield $72 \%$, IR: $\left(v_{\max } \mathrm{cm}^{-1}\right) 1548\left(v_{\mathrm{C}-\mathrm{C}}\right), 750\left(v_{\mathrm{C}-\mathrm{S}}\right), 1645\left(v_{\mathrm{N}=\mathrm{C}}\right), 1438\left(v_{\mathrm{C}-\mathrm{N}}\right)$, $2952\left(v_{\mathrm{C}-\mathrm{H}}\right), 1320\left(v_{\mathrm{N}-\mathrm{N}}\right), 551\left(v_{\mathrm{C}-\mathrm{Br}}\right),{ }^{1} \mathrm{H}$ NMR: $\delta(\mathrm{ppm}) 1.21\left(3 \mathrm{H}, \mathrm{t}, \mathrm{J}=7.3 \mathrm{~Hz}, \mathrm{CH}_{3}\right), 3.08(2 \mathrm{H}$, $\left.\mathrm{q}, \mathrm{J}=7.3 \mathrm{~Hz}, \mathrm{CH}_{2}\right), 7.45-7.96(4 \mathrm{H}, \mathrm{m}, \mathrm{Ar}-\mathrm{H}), 9.42(1 \mathrm{H}, \mathrm{s}, \mathrm{N}=\mathrm{CH}),{ }^{13} \mathrm{C} \mathrm{NMR}: \delta(\mathrm{ppm}) 12.75$ $\left(\mathrm{CH}_{3}\right.$ acyclic), $27.93\left(\mathrm{CH}_{2}\right.$ acyclic),128.41-133.19 (C of aromatic ring), 159.41( $\mathrm{N}=\mathrm{CH}$ acyclic), 156.4,158.7 $\left(\mathrm{C}_{2}, \mathrm{C}_{5}\right.$ of thiadiazole), Anal. Calcd. for: $\mathrm{C}_{11} \mathrm{H}_{10} \mathrm{Br} \mathrm{N} \mathrm{N}_{3} \mathrm{~S}: \mathrm{C}, 44.61, \mathrm{H}$, 3.40, N, $14.19 \%$, found C, 44.21, H, 3.27, N, 14.15\%, Mass $294.96\left(\mathrm{M}^{+}\right)$.

\section{4-Bromo-benzylidene)-(5-ethyl-[1,3,4]thiadiazol-2-yl)-amine (1f)}

M.P.182-183 ${ }^{0}$ C,Yield 68\%, IR: $\left(v_{\max } \mathrm{cm}^{-1}\right)$ 1550( $\left.v_{\mathrm{C}-\mathrm{C}}\right), 752\left(v_{\mathrm{C}-\mathrm{S}}\right), 1660\left(v_{\mathrm{N}=\mathrm{C}}\right), 1441\left(v_{\mathrm{C}-\mathrm{N}}\right)$, $3072\left(v_{\mathrm{C}-\mathrm{H}}\right), 1316\left(v_{\mathrm{N}-\mathrm{N}}\right), 549\left(v_{\mathrm{C}-\mathrm{Br}}\right),{ }^{1} \mathrm{H}$ NMR: $\delta(\mathrm{ppm}) 1.26\left(3 \mathrm{H}, \mathrm{t}, \mathrm{J}=7.3 \mathrm{~Hz}, \mathrm{CH}_{3}\right), 3.06(2 \mathrm{H}$, $\left.\mathrm{q}, \mathrm{J}=7.3 \mathrm{~Hz} \mathrm{CH}_{2}\right), 7.67-7.99(4 \mathrm{H}, \mathrm{m}, \mathrm{Ar}-\mathrm{H}), 9.41(1 \mathrm{H}, \mathrm{s}, \mathrm{N}=\mathrm{CH}),{ }^{13} \mathrm{C} \mathrm{NMR}: \delta(\mathrm{ppm}) 13.76$ $\left(\mathrm{CH}_{3}\right.$ acyclic), $29.73\left(\mathrm{CH}_{2}\right.$ acyclic), 124.09-134.59 (C of aromatic ring), $158.92(\mathrm{~N}=\mathrm{CH}$ acyclic), 158.4,159.9 $\left(\mathrm{C}_{2}, \mathrm{C}_{5}\right.$ of thiadiazole ring), Anal. Calcd for: $\mathrm{C}_{11} \mathrm{H}_{10} \mathrm{Br} \mathrm{N} \mathrm{N}_{3} \mathrm{~S}: \mathrm{C}, 44.61$, $\mathrm{H}, 3.40, \mathrm{~N}, 14.19 \%$, found C, 44.21, H, 3.19, N, $14.11 \%$, Mass 294.98( $\left.\mathrm{M}^{+}\right)$.

\section{2-Nitro-benzylidene)-(5-ethyl-[1,3,4]thiadiazol-2-yl)-amine (1g)}

M.P.176- $179^{0} \mathrm{C}$, Yield $80 \%$, IR: $\left(v \max \mathrm{cm}^{-1}\right)$ 1552( $\left.v_{\mathrm{C}-\mathrm{C}}\right), 753\left(v_{\mathrm{C}-\mathrm{S}}\right), 1649\left(v_{\mathrm{N}=\mathrm{C}}\right), 1436\left(v_{\mathrm{C}-}\right.$ $\left.{ }_{\mathrm{N}}\right), 3090\left(v_{\mathrm{C}-\mathrm{H}}\right), 1321\left(v_{\mathrm{N}-\mathrm{N}}\right), 1518(\mathrm{vC}-\mathrm{NO} 2){ }^{1} \mathrm{H} \mathrm{NMR}: \delta(\mathrm{ppm}) 1.21\left(3 \mathrm{H}, \mathrm{t}, \mathrm{J}=7.1 \mathrm{~Hz}, \mathrm{CH}_{3}\right)$, $3.06\left(2 \mathrm{H}, \mathrm{q}, \mathrm{J}=7.1 \mathrm{~Hz}, \mathrm{CH}_{2}\right), 7.29-7.89(4 \mathrm{H}, \mathrm{m}, \mathrm{Ar}-\mathrm{H}$, $) 9.39(1 \mathrm{H}, \mathrm{s}, \mathrm{N}=\mathrm{CH}),{ }^{13} \mathrm{C} \mathrm{NMR}: \delta$ (ppm) $12.55\left(\mathrm{CH}_{3}\right.$ acyclic), $26.53\left(\mathrm{CH}_{2}\right.$ acyclic), 127.4.-148.40 (C of aromatic ring), $159.10(\mathrm{~N}=\mathrm{CH}$ acyclic $), 157.4,159.8\left(\mathrm{C}_{2}, \mathrm{C}_{5}\right.$ of thiadiazole ring $)$. Anal. Calcd for: $\mathrm{C}_{11} \mathrm{H}_{10} \mathrm{~N}_{4}$ $\mathrm{O}_{2} \mathrm{~S}: \mathrm{C}, 50.37, \mathrm{H}, 3.84 \mathrm{~N}, 21.36 \%$, found $\mathrm{C}, 50.22, \mathrm{H}, 3.59, \mathrm{~N}, 21.16 \%$, Mass $273.06\left(\mathrm{M}^{+}\right)$. 
3-Nitro-benzylidene)-(5-ethyl-[1,3,4]thiadiazol-2-yl)-amine (1h)

M.P.175-177 ${ }^{0}$ C, Yield $79 \%$,IR: $\left(v_{\max } \mathrm{cm}^{-1}\right) 1549\left(v_{\mathrm{C}-\mathrm{C}}\right), 755\left(v_{\mathrm{C}-\mathrm{S}}\right), 1647\left(v_{\mathrm{N}=\mathrm{C}}\right), 1436\left(v_{\mathrm{C}-\mathrm{N}}\right)$, $3110\left(v_{\mathrm{C}-\mathrm{H}}\right), 1322\left(v_{\mathrm{N}-\mathrm{N}}\right), 1521(v \mathrm{C}-\mathrm{NO} 2),{ }^{1} \mathrm{H}$ NMR: $\delta(\mathrm{ppm}) 1.25\left(3 \mathrm{H}, \mathrm{t}, \mathrm{J}=7.4 \mathrm{~Hz}, \mathrm{CH}_{3}\right), 3.07$ $\left(2 \mathrm{H}, \mathrm{q}, \mathrm{J}=7.4 \mathrm{~Hz}, \mathrm{CH}_{2}\right), 7.33-7.83(4 \mathrm{H}, \mathrm{m}, \mathrm{Ar}-\mathrm{H}), 9.41(1 \mathrm{H}, \mathrm{s}, \mathrm{N}=\mathrm{CH}),{ }^{13} \mathrm{C} \mathrm{NMR}: \delta(\mathrm{ppm})$ $14.75\left(\mathrm{CH}_{3}\right.$ acyclic $), 29.93\left(\mathrm{CH}_{2}\right.$ acyclic $), 159.71(\mathrm{~N}=\mathrm{CH}$ acyclic), 117.1- 140.50 (C of aromatic ring), 156.4,159.6 $\left(\mathrm{C}_{2}, \mathrm{C}_{5}\right.$ of thiadiazole ring), Anal. Calcd. For: $\mathrm{C}_{11} \mathrm{H}_{10} \mathrm{~N}_{4} \mathrm{O}_{2} \mathrm{~S}: \mathrm{C}$, 50.37, H, 3.84 N, $21.36 \%$, found $\mathrm{C}, 50.32, \mathrm{H}, 3.49, \mathrm{~N}, 21.14 \%$, Mass $273.05\left(\mathrm{M}^{+}\right)$.

4-Nitro-benzylidene)-(5-ethyl-[1,3,4]thiadiazol-2-yl)-amine (1i)

M.P.176- $178^{0} \mathrm{C}$, Yield $81 \%$, IR $\left.\otimes v_{\max } \mathrm{cm}^{-1}\right) \quad 1550\left(v_{\mathrm{C}-\mathrm{C}}\right), 754\left(v_{\mathrm{C}-\mathrm{S}}\right), 1648\left(v_{\mathrm{N}=\mathrm{C}}\right), 1433\left(v_{\mathrm{C}-}\right.$ $\mathrm{N}), 3120\left(v_{\mathrm{C}-\mathrm{H}}\right), 1317\left(v_{\mathrm{N}-\mathrm{N}}\right), 1526(v \mathrm{C}-\mathrm{NO} 2),{ }^{1} \mathrm{H} \mathrm{NMR}: \delta(\mathrm{ppm}) 1.27\left(3 \mathrm{H}, \mathrm{t}, \mathrm{J}=7.1 \mathrm{~Hz}, \mathrm{CH}_{3}\right)$, $3.08\left(2 \mathrm{H}, \mathrm{q}, \mathrm{J}=7.1 \mathrm{~Hz} \mathrm{CH} \mathrm{CH}_{2}\right), 7.39-7.95(4 \mathrm{H}, \mathrm{m}, \mathrm{Ar}-\mathrm{H}), 9.46(1 \mathrm{H}, \mathrm{s}, \mathrm{N}=\mathrm{CH}) .{ }^{13} \mathrm{C} \delta(\mathrm{ppm})$ $11.85\left(\mathrm{CH}_{3}\right.$ acyclic, $) 26.93\left(\mathrm{CH}_{2}\right.$ acyclic $), 159.21(\mathrm{~N}=\mathrm{CH}$ acyclic $), 117.1-140.30(\mathrm{C}$ of aromatic ring), 157.7,158.6 $\left(\mathrm{C}_{2}, \mathrm{C}_{5}\right.$ of thidiazole ring).Anal. Calcd. For: $\mathrm{C}_{11} \mathrm{H}_{10} \mathrm{~N}_{4} \mathrm{O}_{2} \mathrm{~S}: \mathrm{C}, 50.37, \mathrm{H}$, $3.84 \mathrm{~N}, 21.36 \%$, found $\mathrm{C}, 50.22, \mathrm{H}, 3.32, \mathrm{~N}, 21.21 \%$, Mass $275.02\left(\mathrm{M}^{+}\right)$.

\section{2-Methoxy-benzylidene)-(5-ethyl-[1,3,4]thiadiazol-2-yl)-amine (1j)}

M.P.136-137 ${ }^{0} \mathrm{C}$, Yield $61 \%, \operatorname{IR}\left(v_{\max } \mathrm{cm}^{-1}\right) 1549\left(v_{\mathrm{C}-\mathrm{C}}\right), 752\left(v_{\mathrm{C}-\mathrm{S}}\right), 1639\left(v_{\mathrm{N}=\mathrm{C}}\right), 1439\left(v_{\mathrm{C}-\mathrm{N}}\right), 3090$ $\left(v_{\mathrm{C}-\mathrm{H}}\right), 1314\left(v_{\mathrm{N}-\mathrm{N}}\right), 2969(v \mathrm{OCH} 3),{ }^{1} \mathrm{H}$ NMR: $\delta(\mathrm{ppm}) 1.21\left(3 \mathrm{H}, \mathrm{t}, \mathrm{J}=7.1 \mathrm{~Hz} \mathrm{CH}_{3}\right), 3.17(2 \mathrm{H}$, $\left.\mathrm{q}, \mathrm{J}=7.1 \mathrm{~Hz} \mathrm{CH}_{2}\right), 3.87\left(3 \mathrm{H}, \mathrm{s}, \mathrm{OCH}_{3}\right), 7.03-7.74(4 \mathrm{H}, \mathrm{m}, \mathrm{Ar}-\mathrm{H}), 9.34(1 \mathrm{H}, \mathrm{s}, \mathrm{N}=\mathrm{CH}),{ }^{13} \mathrm{C}$ NMR: $\delta(\mathrm{ppm}) 13.05\left(\mathrm{CH}_{3}\right.$ acyclic), $28.93\left(\mathrm{CH}_{2}\right.$ acyclic), $55.89\left(\mathrm{OCH}_{3}\right), 119.2-159.59(\mathrm{C}$ of aromatic ring), $159.52\left(\mathrm{~N}=\mathrm{CH}\right.$ acyclic), $156.7,159.5\left(\mathrm{C}_{2}, \mathrm{C}_{5}\right.$ of thidiazole). Anal. Calcd for: $\mathrm{C}_{12} \mathrm{H}_{13} \mathrm{~N}_{3}$ OS: C, 58.28, H, $5.30 \mathrm{~N}, 16.99$, \%, found C, 58.12, H, 5.18, N, $16.91 \%$, Mass $258.05\left(\mathrm{M}^{+}\right)$.

\section{3-Methoxy-benzylidene)-(5-ethyl-[1,3,4]thiadiazol-2-yl)-amine (1k)}

M.P.134-136 C, Yield 59\%, IR: $\left(v_{\max } \mathrm{cm}^{-1}\right) 1549\left(v_{\mathrm{C}-\mathrm{C}}\right), 755\left(v_{\mathrm{C}-\mathrm{S}}\right), 1651\left(v_{\mathrm{C}=\mathrm{N}}\right), 1440\left(v_{\mathrm{C}-\mathrm{N}}\right)$, $3085\left(v_{\mathrm{C}-\mathrm{H}}\right), 1314\left(v_{\mathrm{N}-\mathrm{N}}\right), 2973\left(v\right.$ OCH3), ${ }^{1} \mathrm{H}$ NMR: $\delta(\mathrm{ppm}) 1.19\left(3 \mathrm{H}, \mathrm{t}, \mathrm{J}=7.0 \mathrm{~Hz} \mathrm{CH}_{3}\right), 2.97$ $(2 \mathrm{H}, \mathrm{q}, \mathrm{J}=7.0 \mathrm{~Hz} \mathrm{CH}), 3.77\left(3 \mathrm{H}, \mathrm{s}, \mathrm{OCH}_{3}\right), 7.06-7.81(4 \mathrm{H}, \mathrm{m}, \mathrm{Ar}-\mathrm{H}) 9.41(1 \mathrm{H}, \mathrm{s}, \mathrm{N}=\mathrm{CH})$, ${ }^{13} \mathrm{C}$ NMR : $\delta(\mathrm{ppm}) 11.95\left(\mathrm{CH}_{3}\right.$ acyclic $), 26.91\left(\mathrm{CH}_{2}\right.$ acyclic $), 55.79\left(\mathrm{OCH}_{3}\right), 111.02-157.69$ (C of aromatic ring), $158.71\left(\mathrm{~N}=\mathrm{CH}\right.$ acyclic), 156.5,159.4 $\left(\mathrm{C}_{2}, \mathrm{C}_{5}\right.$ of thiadiazole). Anal. Calcd. For: $\mathrm{C}_{12} \mathrm{H}_{13} \mathrm{~N}_{3}$ OS: C, 58.28, H,5 .30 N,16.99 \%, found C, 58.12, H, .16, N, $16.81 \%$, Mass $258.05\left(\mathrm{M}^{+}\right)$.

4-Methoxy-benzylidene)-(5-ethyl-[1,3,4]thiadiazol-2-yl)-amine (1l)

M.P.136-138 ${ }^{0} \mathrm{C}$, Yield $57 \%$,IR: $\left(v_{\max } \mathrm{cm}^{-1}\right) 1549\left(v_{\mathrm{C}-\mathrm{C}}\right), 755\left(v_{\mathrm{C}-\mathrm{S}}\right), 1648\left(v_{\mathrm{C}=\mathrm{N}}\right), 1441\left(v_{\mathrm{C}-\mathrm{N}}\right)$, $3095\left(v_{\mathrm{C}-\mathrm{H}}\right), 1314\left(v_{\mathrm{N}-\mathrm{N}}\right), 1109\left(v_{\mathrm{OCH}}\right),{ }^{1} \mathrm{H}$ NMR: $\delta(\mathrm{ppm}) 1.26\left(3 \mathrm{H}, \mathrm{t}, \mathrm{J}=7.1 \mathrm{~Hz}, \mathrm{CH}_{3}\right)$, $3.17\left(2 \mathrm{H}, \mathrm{q}, \mathrm{J}=7.1 \mathrm{~Hz} \mathrm{CH}_{2}\right), 3.84\left(3 \mathrm{H}, \mathrm{s}, \mathrm{OCH}_{3}\right), 7.26-7.73(4 \mathrm{H}, \mathrm{m}, \mathrm{Ar}-\mathrm{H}), 9.35(1 \mathrm{H}, \mathrm{s}$, $\mathrm{N}=\mathrm{CH}),{ }^{13} \mathrm{C}$ NMR: $\delta \quad(\mathrm{ppm}) 11.95\left(\mathrm{CH}_{3}\right.$ acyclic $), 26.98\left(\mathrm{CH}_{2}\right.$ acyclic $), 55.51\left(\mathrm{OCH}_{3}\right), 114.5-$ 160.40 (C of aromatic ring), $158.61\left(\mathrm{~N}=\mathrm{CH}\right.$ acyclic), 160.4,156.7 $\left(\mathrm{C}_{2}, \mathrm{C}_{5}\right.$ of thiadiazole). Anal. Calcd for: $\mathrm{C}_{12} \mathrm{H}_{13} \mathrm{~N}_{3}$ OS: C, 58.28, H, $5.30 \mathrm{~N}, 16.99$, \%, found C, 58.12, H, 5.21, N, $16.11 \%$, Mass $258.03\left(\mathrm{M}^{+}\right)$.

\section{Synthesis of 3-chloro-1-(5-ethyl-[1,3,4]thiadiazol-2-yl)-4-phenyl-azetidin-2-one (2)}

Equimolar reaction of compound $\mathbf{1}\left(0.004\right.$ mole) and $\mathrm{ClCH}_{2} \mathrm{COCl}(0.004$ mole) in the presence of triethylamine (0.004 mole). In the reaction solution $\mathrm{ClCH}_{2} \mathrm{COCl}$ was added drop wise at -5 ${ }^{0} \mathrm{C}$ temperature in crushed ice container. After the reaction mixture was stirred for about $2 \mathrm{~h}$ then followed by heating to reflux on steam bath for $4 \mathrm{~h}$ and checked the reaction progress using TLC at various stages. After completion of reaction the container (R.B. Flask) was 
removed from steam bath and excess solvent distilled off and the obtained solid product creamy white crystal which was purified over a silica gel column using chloroform: methanol $(8: 2 \mathrm{v} / \mathrm{v})$ mixture as eluant was recrystallized from ethanol at room temperature to yielded compound 2 (Figure 2). White crystalline solid, M.P. $189-191{ }^{\circ} \mathrm{C}$, yield $72 \%$, IR $\left(v_{\max } \mathrm{cm}^{-1}\right) 1453\left(v_{\mathrm{C}-\mathrm{C}}\right), 714$ $\left(v_{\mathrm{C}-\mathrm{S}}\right), 1667\left(v_{\mathrm{C}=\mathrm{N}}\right), 1740\left(v_{\mathrm{C}=\mathrm{O}}\right.$, cyclic azetidin-2-one $), 1332\left(v_{\mathrm{C}-\mathrm{N}}\right), 3118\left(v_{\mathrm{C}-\mathrm{H}}\right), 2917\left(\mathrm{~V}_{\mathrm{CH}-\mathrm{Cl}}\right),{ }^{1} \mathrm{H}$ NMR: $\delta(\mathrm{ppm}) 1.06\left(3 \mathrm{H}, \mathrm{t}, \mathrm{J}=7.3 \mathrm{~Hz}, \mathrm{CH}_{3}\right), 2.86\left(2 \mathrm{H}, 2.80\left(\mathrm{q}, \mathrm{J}=7.3 \mathrm{~Hz}, \mathrm{CH}_{2}\right), 5.16(1 \mathrm{H}, \mathrm{d}, \mathrm{J}=\right.$ $5.5 \mathrm{~Hz}, \mathrm{~N}-\mathrm{C}-\mathrm{H}$ azetidin-2-one), $5.73(1 \mathrm{H}, \mathrm{d}, \mathrm{J}=5.5 \mathrm{~Hz}, \mathrm{Cl}-\mathrm{CH}$ azetidin-2-one), 7.24-7.35 (5H, m,Ar-H), ${ }^{13} \mathrm{C}$ NMR: $\delta$ (ppm) $12.55\left(\mathrm{CH}_{3}\right.$ acyclic), 27.13( $\mathrm{CH}_{2}$ acyclic), 127.0-139.0 (C of aromatic ring),58.1(CH azetidin-2-one),59.5(CH-Clazetidin-2-one), 161.3, $156.8\left(\mathrm{C}_{2}, \mathrm{C}_{5}\right.$ of thiadiazole ring), $167.5\left(\mathrm{C}=\mathrm{O}\right.$ cyclic azetidin-2-one), Anal. Calcd for: $\mathrm{C}_{13} \mathrm{H}_{12} \mathrm{~N}_{3} \mathrm{ClOS}$ : C, 53.15, $\mathrm{H}, 4.12 \mathrm{~N}, 14.30 \%$, found C, 53.02, H, 4.08, N, $1415 \%$, Mass $293.05\left(\mathrm{M}^{+}\right)$

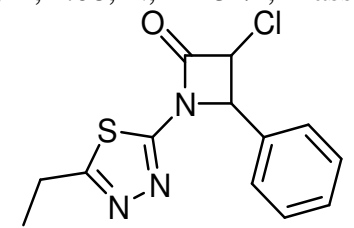

Figure 2. Structure of Compound 2

The compounds $\mathbf{2} \boldsymbol{a}-\mathbf{2} \boldsymbol{l}$ were synthesized by the similar method as reported earlier 3-Chloro-4-(2-chloro-phenyl)-1-(5-ethyl-[1,3,4]thiadiazol-2-yl)-azetidin-2-one (2a) M.P. $189-191{ }^{0} \mathrm{C}$, Yield $72 \%$, IR: $\left(v_{\max } \mathrm{cm}^{-1}\right) 1451\left(v_{\mathrm{C}-\mathrm{C}}\right), 713\left(v_{\mathrm{C}-\mathrm{S}}\right), 1670\left(v_{\mathrm{C}=\mathrm{N}}\right), 1741$ $\left(v_{\mathrm{C}=\mathrm{O}}\right.$ Cyclic azetidin-2-one $), 1430\left(v_{\mathrm{C}-\mathrm{N}}\right), 3118\left(v_{\mathrm{C}-\mathrm{H}}\right), 1310\left(v_{\mathrm{N}-\mathrm{N}}\right), 2917\left(v_{\mathrm{CH}-\mathrm{Cl}}\right),{ }^{1} \mathrm{H}$ NMR: $\delta$ (ppm) $1.09\left(3 \mathrm{H}, \mathrm{t}, \mathrm{J}=7.3 \mathrm{~Hz}, \mathrm{CH}_{3}\right), 2.85\left(2 \mathrm{H}, \mathrm{q}, \mathrm{J}=7.3 \mathrm{~Hz}, \mathrm{CH}_{2}\right), 5.18(1 \mathrm{H}, \mathrm{d}, \mathrm{J}=5.5 \mathrm{HzN}-$ $\mathrm{C}-\mathrm{H}$ azetidin-2-one), $5.79(1 \mathrm{H}, \mathrm{d}, \mathrm{J}=5.5 \mathrm{HzCl}-\mathrm{CH}$ azetidin-2-one), 7.36-7.56 (4H, m, Ar-H), ${ }^{13} \mathrm{C}$ NMR: $\delta(\mathrm{ppm}) 12.25\left(\mathrm{CH}_{3}\right.$ acyclic), 27.23( $\mathrm{CH}_{2}$ acyclic), 127.4-137.3 (C of aromatic ring), 58.6(CHazetidin-2-one),59.9(CH-Clazetidin-2-one), 160.3,157.6( $\mathrm{C}_{2}, \mathrm{C}_{5}$ of thiadiazole ring), 168.0(C=O Cyclic azetidin-2-one), Anal. Calcd. for: $\mathrm{C}_{13} \mathrm{H}_{11} \mathrm{~N}_{3} \mathrm{Cl}_{2} \mathrm{OS}: \mathrm{C}, 47.57, \mathrm{H}, 3.38$ $\mathrm{N}, 12.80 \%$, found $\mathrm{C}, 47.20, \mathrm{H}, 3.15, \mathrm{~N}, 12.61 \%$, Mass $327.00\left(\mathrm{M}^{+}\right)$.

3-Chloro-4-(3-chloro-phenyl)-1-(5-ethyl-[1,3,4]thiadiazol-2-yl)-azetidin-2-one (2b) M.P.172-175 ${ }^{0} \mathrm{C}$, Yield $66 \%$, IR: $\left(v_{\max } \mathrm{cm}^{-1}\right) 1543\left(v_{\mathrm{C}-\mathrm{C}}\right), 752\left(v_{\mathrm{C}-\mathrm{S}}\right), 1655\left(v_{\mathrm{C}=\mathrm{N}}\right), 1432\left(v_{\mathrm{C}-\mathrm{N}}\right)$, $3018\left(v_{\mathrm{C}-\mathrm{H}}\right), 1310\left(v_{\mathrm{N}-\mathrm{N}}\right), 2919\left(v_{\mathrm{CH}-\mathrm{Cl}}\right), 1739\left(v_{\mathrm{C}=\mathrm{O}}\right) \mathrm{C}=\mathrm{O}$ cyclic azetidin-2-one, ${ }^{1} \mathrm{H}$ NMR: $\delta(\mathrm{ppm}) 1.10\left(3 \mathrm{H}, \mathrm{t}, \mathrm{J}=7.3 \mathrm{HzCH}_{3}\right), 2.84\left(2 \mathrm{H}, \mathrm{q}, \mathrm{J}=7.3 \mathrm{~Hz}, \mathrm{CH}_{2}\right), 5.68(1 \mathrm{H}, \mathrm{d}, \mathrm{J}=5.5 \mathrm{HzN}-$ $\mathrm{Cl}-\mathrm{CH}$ azetidin-2-one), $5.29(1 \mathrm{H}, \mathrm{d}, \mathrm{J}=5.5 \mathrm{~Hz}, \mathrm{NCH}$ azetidin-2-one), 7.26-7.62 (4H, m,Ar$\mathrm{H}),{ }^{13} \mathrm{C} \mathrm{NMR}: \delta(\mathrm{ppm}) 12.27\left(\mathrm{CH}_{3}\right.$ acyclic), 28.03 $\left(\mathrm{CH}_{2}\right.$ acyclic),127.0-139.3 (C of aromatic ring), 58.1(CHazetidin-2-one),59.9(C-Clazetidin-2-one),161.1,157.3( $\mathrm{C}_{2}, \mathrm{C}_{5}$ of thiadiazole), 167.5(C $=\mathrm{O}$ cyclic azetidin-2-one), Anal. Calcd for: $\mathrm{C}_{13} \mathrm{H}_{11} \mathrm{~N}_{3} \mathrm{Cl}_{2} \mathrm{OS}$ : C, 47.57, H,3.38 $\mathrm{N}, 12.80 \%$, found $\mathrm{C}, 47.32, \mathrm{H}, 3.22, \mathrm{~N}, 12.71 \%$, Mass $327.01\left(\mathrm{M}^{+}\right)$.

3-Chloro-4-(4-chloro-phenyl)-1-(5-ethyl-[1,3,4]thiadiazol-2-yl)-azetidin-2-one (2c)

M.P.173-175 ${ }^{0} \mathrm{C}$, Yield $67 \%$, IR: $\left(v_{\max } \mathrm{cm}^{-1}\right) 1549\left(v_{\mathrm{C}-\mathrm{C}}\right), 746\left(v_{\mathrm{C}-\mathrm{S}}\right), 1661\left(v_{\mathrm{C}=\mathrm{N}}\right), 1439\left(v_{\mathrm{C}-\mathrm{N}}\right)$, $3108\left(v_{\mathrm{C}-\mathrm{H}}\right), 1316\left(v_{\mathrm{N}-\mathrm{N}}\right), 2919\left(v_{\mathrm{CH}-\mathrm{Cl}}\right), 1740\left(v_{\mathrm{C}=\mathrm{O}}\right.$ cyclic azetidin-2-one $),{ }^{1} \mathrm{H}$ NMR: $\delta(\mathrm{ppm}) 1.07$ $\left(3 \mathrm{H}, \mathrm{t}, \mathrm{J}=7.3 \mathrm{~Hz}, \mathrm{CH}_{3}\right), 2.80\left(2 \mathrm{H}, \mathrm{q}, \mathrm{J}=7.3 \mathrm{~Hz}, \mathrm{CH}_{2}\right), 5.17(1 \mathrm{H}, \mathrm{d}, \mathrm{J}=5.5 \mathrm{HzN}-\mathrm{C}-\mathrm{H}$ azetidin-2-one), $5.75(1 \mathrm{H}, \mathrm{d}, \mathrm{J}=5.5 \mathrm{~Hz}, \mathrm{Cl}-\mathrm{CH}$ azetidin-2-one), 7.52-7.66 (4H, m,Ar-H), ${ }^{13} \mathrm{C}$ NMR $: \delta(\mathrm{ppm}) 12.25\left(\mathrm{CH}_{3}\right.$ acyclic), $28.23\left(\mathrm{CH}_{2}\right.$ acyclic), 129.4-139.4 (C of aromatic ring), 58.6(CHazetidin-2-one),59.5(C-Cl azetidin-2-one), 160.3, $157.8\left(\mathrm{C}_{2}, \mathrm{C}_{5}\right.$ of thiadiazole ring), 168.2( $\mathrm{C}=\mathrm{O}$ Cyclic azetidin-2-one), Anal. CalcdFor: $\mathrm{C}_{13} \mathrm{H}_{11} \mathrm{~N}_{3} \mathrm{Cl}_{2} \mathrm{OS} \mathrm{C}, 47.57, \mathrm{H}$, $3.38 \mathrm{~N}, 12.80 \%$, found $\mathrm{C}, 47.27, \mathrm{H}, 3.16, \mathrm{~N}, 11.61 \%$, Mass $327.00\left(\mathrm{M}^{+}\right)$ 
3-Chloro-4-(2-bromo-phenyl)-1-(5-ethyl-[1,3,4]thiadiazol-2-yl)-azetidin-2-one (2d) M.P.178-180 C, Yield $71 \%$, IR: $\left(v_{\max } \mathrm{cm}^{-1}\right) 1546\left(v_{\mathrm{C}-\mathrm{C}}\right), 741\left(v_{\mathrm{C}-\mathrm{S}}\right), 1645\left(v_{\mathrm{C}=\mathrm{N}}\right), 1442\left(v_{\mathrm{C}-\mathrm{N}}\right)$, $2959\left(v_{\mathrm{C}-\mathrm{H}}\right), 1325\left(v_{\mathrm{N}-\mathrm{N}}\right), \quad 549\left(v_{\mathrm{C}-\mathrm{Br}}\right) \mathrm{Ar}, 1738\left(v_{\mathrm{C}=\mathrm{O}}\right.$ cyclic azetidin-2-one $), 2918\left(\mathrm{~V}_{\mathrm{CH}-\mathrm{Cl}}\right),{ }^{1} \mathrm{H}$ NMR: $\delta(\mathrm{ppm}) 1.06\left(3 \mathrm{H}, \mathrm{t}, \mathrm{J}=7.3 \mathrm{~Hz}, \mathrm{CH}_{3}\right), 2.80\left(2 \mathrm{H}, \mathrm{q}, \mathrm{J}=7.3 \mathrm{~Hz}, \mathrm{CH}_{2}\right), 5.22(1 \mathrm{H}, \mathrm{d}, \mathrm{J}=$ $5.5 \mathrm{~Hz}, \mathrm{~N}-\mathrm{C}-\mathrm{H}$ azetidin-2-one), $5.75(1 \mathrm{H}, \mathrm{d}, \mathrm{J}=5.5 \mathrm{HzCl}-\mathrm{CH}$ azetidin-2-one $), 6.72-7.82$ $(4 \mathrm{H}, \mathrm{m}, \mathrm{Ar}-\mathrm{H}),{ }^{13} \mathrm{C} \mathrm{NMR}: \delta(\mathrm{ppm}) 12.29\left(\mathrm{CH}_{3}\right.$ acyclic $), 27.23\left(\mathrm{CH}_{2}\right.$ acyclic $), 128.9-139.3(\mathrm{C}$ of aromatic ring), 58.8(CHazetidin-2-one), 59.9 (C-Clazetidin-2-one), 161.6, $157.1 \quad\left(\mathrm{C}_{2}, \mathrm{C}_{5}\right.$ of thiadiazole ring), 168.3( $\mathrm{C}=\mathrm{O}$ cyclic azetidin-2-one), Anal. Calcd for: $\mathrm{C}_{13} \mathrm{H}_{11} \mathrm{~N}_{3}$ BrClOS: C, 41.90, H,2.98 N,11.28\%, found C, 41.07, H, 2.70, N, $11.21 \%$, Mass 370.95( $\left.\mathrm{M}^{+}\right)$.

3-Chloro-4-(3-bromo-phenyl)-1-(5-ethyl-[1,3,4]thiadiazol-2-yl)-azetidin-2-one (2e)

M.P. $180-182^{\circ} \mathrm{C}$, yield $75 \%$, IR: $\left(v_{\max } \mathrm{cm}^{-1}\right)-1548\left(v_{\mathrm{C}-\mathrm{C}}\right), 750\left(v_{\mathrm{C}-\mathrm{S}}\right), 1645\left(v_{\mathrm{C}=\mathrm{N}}\right), 1438\left(v_{\mathrm{C}-\mathrm{N}}\right)$, $2952\left(v_{\mathrm{C}-\mathrm{H}}\right), 1320\left(v_{\mathrm{N}-\mathrm{N}}\right), 551\left(v_{\mathrm{C}-\mathrm{Br}}\right) \mathrm{Ar}, 1741\left(v_{\mathrm{C}=\mathrm{O}}\right.$ cyclic azetidin-2-one $), 2917(\mathrm{vCH}-\mathrm{Cl}),{ }^{1} \mathrm{H}$ NMR: $\delta(\mathrm{ppm}) 1.09\left(3 \mathrm{H}, \mathrm{t}, \mathrm{J}=7.3 \mathrm{~Hz}, \mathrm{CH}_{3}\right), 2.81\left(2 \mathrm{H}, \mathrm{q}, \mathrm{J}=7.3 \mathrm{~Hz}, \mathrm{CH}_{2}\right), 5.19(1 \mathrm{H}, \mathrm{d}, \mathrm{J}=$ $5.5 \mathrm{~Hz}, \mathrm{~N}-\mathrm{C}-\mathrm{H}$ azetidin-2-one), $5.70(1 \mathrm{H}, \mathrm{d}, \mathrm{J}=5.5 \mathrm{~Hz}, \mathrm{Cl}-\mathrm{CH}$ azetidin-2-one), 7.04-7.45 $(4 \mathrm{H}, \mathrm{m}, \mathrm{Ar}-\mathrm{H}),{ }^{13} \mathrm{C}$ NMR: $\delta(\mathrm{ppm}) 12.21\left(\mathrm{CH}_{3}\right.$ acyclic), 28.03( $\mathrm{CH}_{2}$ acyclic $), 118.2-139.3(\mathrm{C}$ of aromatic ring), 57.9(Chazetidin-2-one), 60.2(CH-Clazetidin-2-one), 160.4,158.3( $\mathrm{C}_{2}, \mathrm{C}_{5}$ of thiadiazole ring), $167.8\left(\mathrm{C}=\mathrm{O}\right.$ cyclic azetidin-2-one), Anal. Calcd for: $\mathrm{C}_{13} \mathrm{H}_{11} \mathrm{BrClN}_{3} \mathrm{OS}: \mathrm{C}$, 41.90, H,2.98 N, $11.28 \%$, found C, 41.17, H, 2.75, N, $11.13 \%$, Mass 370.92( $\left.\mathrm{M}^{+}\right)$.

\section{3-Chloro-4-(4-bromo-phenyl)-1-(5-ethyl-[1,3,4]thiadiazol-2-yl)-azetidin-2-one (2f)}

M.P.180-182 C, Yield $68 \%$, IR: $\left(v_{\max } \mathrm{cm}^{-1}\right)$ 1551( $\left.v_{\mathrm{C}-\mathrm{C}}\right), 752\left(v_{\mathrm{C}-\mathrm{S}}\right), 1668\left(v_{\mathrm{C}=\mathrm{N}}\right), 1441\left(v_{\mathrm{C}-\mathrm{N}}\right)$, $3072\left(v_{\mathrm{C}-\mathrm{H}}\right), 1326\left(v_{\mathrm{N}-\mathrm{N}}\right), 549\left(v_{\mathrm{C}-\mathrm{Br}}\right) \mathrm{Ar}, 1739\left(v_{\mathrm{C}=\mathrm{O}}\right.$ cyclic azetidin-2-one $), 2920\left(\mathrm{~V}_{\mathrm{CH}-\mathrm{Cl}}\right),{ }^{1} \mathrm{H}$ NMR: $\delta(\mathrm{ppm}) 1.11\left(3 \mathrm{H}, \mathrm{t}, \mathrm{J}=7.3 \mathrm{~Hz}, \mathrm{CH}_{3}\right), 2.80\left(2 \mathrm{H}, \mathrm{q}, \mathrm{J}=7.3 \mathrm{~Hz}, \mathrm{CH}_{2}\right), 5.21(1 \mathrm{H}, \mathrm{d}, \mathrm{J}=5.5$ $\mathrm{Hz}, \mathrm{N}-\mathrm{C}-\mathrm{H}$ azetidin-2-one), $5.76(1 \mathrm{H}, \mathrm{d}, \mathrm{J}=5.5 \mathrm{~Hz}, \mathrm{Cl}-\mathrm{CH}$ azetidin-2-one), 6.96-7.350 (4H, m,Ar-H), ${ }^{13} \mathrm{C}$ NMR : $\delta(\mathrm{ppm}) 12.11\left(\mathrm{CH}_{3}\right.$ acyclic $), 27.05\left(\mathrm{CH}_{2}\right.$ acyclic $), 124.2-139.5(\mathrm{C}$ of aromatic ring), 58.6 (Chazetidin-2-one), 60.0 (CH-Clazetidin-2-one), 159.4,157.8 $\left(\mathrm{C}_{2}, \mathrm{C}_{5}\right.$ of thiadiazole ring), $169.8\left(\mathrm{C}=\mathrm{O}\right.$ cyclic azetidin-2-one), Anal. Calcd for: $\mathrm{C}_{13} \mathrm{H}_{11} \mathrm{BrClN}_{3} \mathrm{OS}$ : $\mathrm{C}$, 41.90, H,2.98 N, $11.28 \%$, found C, 41.77, H, 2.79, N, 11.19\%, Mass 370.91( $\left.\mathrm{M}^{+}\right)$.

\section{3-Chloro-4-(2-Nitro-phenyl)-1-(5-ethyl-[1,3,4]thiadiazol-2-yl)-azetidin-2-one (2g)}

M.P.176-179 ${ }^{0}$, yield $80 \%$, IR: $\left(v_{\max } \mathrm{cm}^{-1}\right)-1572\left(v_{\mathrm{C}-\mathrm{C}}\right), 753\left(v_{\mathrm{C}-\mathrm{S}}\right), 1649\left(v_{\mathrm{C}=\mathrm{N}}\right), 1436\left(v_{\mathrm{C}-\mathrm{N}}\right)$, $3040\left(v_{\mathrm{C}-\mathrm{H}}\right), 1321\left(v_{\mathrm{N}-\mathrm{N}}\right), 1516\left(\mathrm{vC}-\mathrm{NO}_{2}\right) \mathrm{Ar}, 1738\left(v_{\mathrm{C}=\mathrm{O}}\right.$ cyclic azetidin-2-one $), 2921\left(v_{\mathrm{CH}-\mathrm{Cl}}\right),{ }^{1} \mathrm{H}$ NMR: $\delta(\mathrm{ppm}) 1.04\left(3 \mathrm{H}, \mathrm{t}, \mathrm{J}=7.3 \mathrm{~Hz}, \mathrm{CH}_{3}\right), 2.80\left(2 \mathrm{H}, \mathrm{q}, \mathrm{J}=7.3 \mathrm{~Hz}, \mathrm{CH}_{2}\right), 5.28(1 \mathrm{H}, \mathrm{d}, \mathrm{J}=$ $5.5 \mathrm{~Hz}, \mathrm{~N}-\mathrm{C}-\mathrm{H}$ azetidin-2-one), $5.94(1 \mathrm{H}, \mathrm{d}, \mathrm{J}=5.5 \mathrm{~Hz}, \mathrm{Cl}-\mathrm{CH}$ azetidin-2-one), 6.96-7.35 $(4 \mathrm{H}, \mathrm{m}, \mathrm{Ar}-\mathrm{H}),{ }^{13} \mathrm{C}$ NMR: $\delta$ (ppm) 12.11( $\mathrm{CH}_{3}$ acyclic), 28.02( $\mathrm{CH}_{2}$ acyclic $), 120.2-148.4(\mathrm{C}$ of aromatic ring), 58.2(Chazetidin-2-one), 59.6(CH-Clazetidin-2-one), 160.2, $158.4 \quad\left(\mathrm{C}_{2}, \mathrm{C}_{5}\right.$ of thiadiazole ring), $168.5\left(\mathrm{C}=\mathrm{O}\right.$ cyclic azetidin-2-one), Anal. Calcd for: $\mathrm{C}_{13} \mathrm{H}_{11} \mathrm{ClN}_{3} \mathrm{O}_{3} \mathrm{~S}: \mathrm{C}$, 46.09, H,3.27, N,16.54 \%, found C, 46.01, H, 3.19, N, $16.15 \%$, Mass 338.02( $\left.\mathrm{M}^{+}\right)$.

\section{3-Chloro-4-(3-Nitro-phenyl)-1-(5-ethyl-[1,3,4]thiadiazol-2-yl)-azetidin-2-one (2h)}

M.P.175-177 ${ }^{0} \mathrm{C}$, Yield $79 \%$, IR: $\left(v_{\max } \mathrm{cm}^{-1}\right) 1549\left(v_{\mathrm{C}-\mathrm{C}}\right), 755\left(v_{\mathrm{C}-\mathrm{S}}\right), 1635\left(v_{\mathrm{C}=\mathrm{N}}\right), 1436\left(v_{\mathrm{C}-\mathrm{N}}\right)$, $3110\left(v_{\mathrm{C}-\mathrm{H}}\right), 1322\left(v_{\mathrm{N}-\mathrm{N}}\right), 1521\left(v \mathrm{C}-\mathrm{NO}_{2}\right) \mathrm{Ar}, 1737\left(v_{\mathrm{C}=\mathrm{O}}\right.$ cyclic azetidin-2-one $), 2922\left(v_{\mathrm{CH}-\mathrm{Cl}}\right),{ }_{1} \mathrm{H}$ NMR: $\delta(\mathrm{ppm}) 1.13\left(3 \mathrm{H}, \mathrm{t}, \mathrm{J}=7.3 \mathrm{~Hz}, \mathrm{CH}_{3}\right), 2.80\left(2 \mathrm{H}, \mathrm{q}, \mathrm{J}=7.3 \mathrm{~Hz}, \mathrm{CH}_{2}\right), 5.37(1 \mathrm{H}, \mathrm{d}, \mathrm{J}=$ $5.5 \mathrm{~Hz}, \mathrm{~N}-\mathrm{C}-\mathrm{H}$ azetidin-2-one), $5.78(1 \mathrm{H}, \mathrm{d}, \mathrm{J}=5.5 \mathrm{~Hz}, \mathrm{Cl}-\mathrm{CH}$ azetidin-2-one), 7.46-8.21 (4H, m,Ar-H $),{ }^{13} \mathrm{C}$ NMR: $\delta$ (ppm) 12.16 $\left(\mathrm{CH}_{3}\right.$ acyclic), 27.02( $\mathrm{CH}_{2}$ acyclic), 116.4-140.5(C of aromatic ring), 58.7(Chazetidin-2-one), 59.4(CH-Clazetidin-2-one),161.2,157.4 $\left(\mathrm{C}_{2}, \mathrm{C}_{5}\right.$ of thiadiazole ring), $168.7\left(\mathrm{C}=\mathrm{O}\right.$ cyclic azetidin-2-one), $\mathrm{C}_{13} \mathrm{H}_{11} \mathrm{ClN}_{3} \mathrm{O}_{3} \mathrm{~S}: \mathrm{C}, 46.09, \quad \mathrm{H}, 3.27$, $\mathrm{N}, 16.54, \%$, found $\mathrm{C}, 46.02, \mathrm{H}, 3.19, \mathrm{~N}, 16.41 \%$, Mass $338.03\left(\mathrm{M}^{+}\right)$. 
3-Chloro-4-(4-Nitro-phenyl)-1-(5-ethyl-[1,3,4]thiadiazol-2-yl)-azetidin-2-one (2i)

M.P. $183-185^{\circ}$ C, Yield $81 \%$, IR: $\left(v_{\max } \mathrm{cm}^{-1}\right) 1542\left(v_{\mathrm{C}-\mathrm{C}}\right), 754\left(v_{\mathrm{C}-\mathrm{S}}\right), 1643\left(v_{\mathrm{C}=\mathrm{N}}\right), 1433\left(v_{\mathrm{C}-}\right.$ $\left.{ }_{\mathrm{N}}\right), 3120\left(v_{\mathrm{C}-\mathrm{H}}\right), 1317\left(v_{\mathrm{N}-\mathrm{N}}\right), 1526\left(\mathrm{vC}-\mathrm{NO}_{2}\right) \mathrm{Ar}, 1740\left(v_{\mathrm{C}=\mathrm{O}}\right.$ cyclic azetidin-2-one $), 2919\left(v_{\mathrm{CH}-\mathrm{Cl}}\right),{ }_{\mathrm{H}} \mathrm{H}$ NMR: $\delta(\mathrm{ppm}) 1.15\left(3 \mathrm{H}, \mathrm{t}, \mathrm{J}=7.3 \mathrm{~Hz}, \mathrm{CH}_{3}\right), 2.82\left(2 \mathrm{H}, \mathrm{q}, \mathrm{J}=7.3 \mathrm{~Hz}, \mathrm{CH}_{2}\right), 5.23(1 \mathrm{H}, \mathrm{d}, \mathrm{J}=$ $5.5 \mathrm{~Hz}, \mathrm{~N}-\mathrm{C}-\mathrm{H}$ azetidin-2-one), $5.85(1 \mathrm{H}, \mathrm{d}, \mathrm{J}=5.5 \mathrm{~Hz}, \mathrm{Cl}-\mathrm{CH}$ azetidin-2-one), 7.25-8.07 (4H, m,Ar-H), ${ }^{13} \mathrm{C}$ NMR: $\delta$ (ppm) $12.96\left(\mathrm{CH}_{3}\right.$ acyclic), 27.07( $\mathrm{CH}_{2}$ acyclic $), 117.4-127.3(\mathrm{C}$ of aromatic ring), 57.9( $\mathrm{CH}$ azetidin-2-one), 58.8 ( $\mathrm{CH}$-Clazetidin-2-one), 162.1, $156.9\left(\mathrm{C}_{2}, \mathrm{C}_{5}\right.$ of thiadiazole ring), $168.9\left(\mathrm{C}=\mathrm{O}\right.$ cyclic azetidin-2-one), Anal. Calcd.for: $\mathrm{C}_{13} \mathrm{H}_{11} \mathrm{ClN}_{3} \mathrm{O}_{3} \mathrm{~S}: \mathrm{C}$, 46.09, H, 3.27, N, $16.54 \%$, found C, 46.02, H, 3.12, N, $16.12 \%$, Mass $338.01\left(\mathrm{M}^{+}\right)$.

3-Chloro-4-(2-methoxy-phenyl)-1-(5-ethyl-[1,3,4]thiadiazol-2-yl)-azetidin-2-one (2j)

M.P.139-141 ${ }^{0} \mathrm{C}$, Yield $64 \%$, IR: $\left(v_{\max } \mathrm{cm}^{-1}\right) 1548\left(v_{\mathrm{C}-\mathrm{C}}\right), 752\left(v_{\mathrm{C}-\mathrm{S}}\right), 1619\left(v_{\mathrm{C}=\mathrm{N}}\right), 1439\left(v_{\mathrm{C}-\mathrm{N}}\right)$, $3090\left(v_{\mathrm{C}-\mathrm{H}}\right), 1314\left(v_{\mathrm{N}-\mathrm{N}}\right), 2969\left(\mathrm{v} \mathrm{OCH}_{3}\right), 1738\left(v_{\mathrm{C}=\mathrm{O}}\right.$ cyclic azetidin-2-one $), 2918\left(v_{\mathrm{CH}-\mathrm{Cl}}\right),{ }^{1} \mathrm{H}$ NMR: $\delta(\mathrm{ppm}) 1.09\left(3 \mathrm{H}, \mathrm{t}, \mathrm{J}=7.3 \mathrm{HzCH}_{3}\right), 2.80\left(2 \mathrm{H}, \mathrm{q}, \mathrm{J}=7.3 \mathrm{~Hz}, \mathrm{CH}_{2}\right), 3.79\left(3 \mathrm{H}, \mathrm{s}, \mathrm{Ar}-\mathrm{OCH}_{3}\right), 5.08$ $(1 \mathrm{H}, \mathrm{d}, \mathrm{J}=5.5 \mathrm{~Hz}, \mathrm{~N}-\mathrm{C}-\mathrm{H}$ azetidin-2-one $), 5.61(1 \mathrm{H}, \mathrm{d}, \mathrm{J}=5.5 \mathrm{~Hz}, \mathrm{Cl}-\mathrm{CH}$ azetidin-2-one $), 6.94-$ $7.22(4 \mathrm{H}, \mathrm{m}, \mathrm{Ar}-\mathrm{H}),{ }^{13} \mathrm{C}$ NMR: $\delta(\mathrm{ppm}) 12.76\left(\mathrm{CH}_{3}\right.$ acyclic $), 27.12\left(\mathrm{CH}_{2}\right.$ acyclic $), 113.7-131.7(\mathrm{C}$ of aromatic ring),55.9 $\left(\mathrm{OCH}_{3}\right), 58.06(\mathrm{CHazetidin}$-2-one),59.5( $\mathrm{CH}$-Clazetidin-2-one), 161.3,156.8 $\left(\mathrm{C}_{2}, \mathrm{C}_{5}\right.$ of thiadiazole ring), $168.3\left(\mathrm{C}=\mathrm{O}\right.$ cyclic azetidin-2-one), Anal. Calcd.for: $\mathrm{C}_{14} \mathrm{H}_{14} \mathrm{ClN}_{3} \mathrm{O}_{2} \mathrm{~S}$ :C, 51.93, H, 4.36, N, 12.98, \%, found C, 51.77, H, 4.19, N, 12.22, \%, Mass 323.05( $\left(^{+}\right)$.

3-Chloro-4-(3-methoxy-phenyl)-1-(5-ethyl-[1,3,4]thiadiazol-2-yl)-azetidin-2-one (2k)

M.P.140-142 ${ }^{0} \mathrm{C}$, Yield $67 \%$, IR: $\left(v_{\max } \mathrm{cm}^{-1}\right)-1549\left(v_{\mathrm{C}-\mathrm{C}}\right), 755\left(v_{\mathrm{C}-\mathrm{S}}\right), 1625\left(v_{\mathrm{C}=\mathrm{N}}\right), 1440\left(v_{\mathrm{C}-\mathrm{N}}\right), 3085$ $\left(v_{\mathrm{C}-\mathrm{H}}\right), 1314\left(v_{\mathrm{N}-\mathrm{N}}\right), 2973\left(\mathrm{vOCH}_{3}\right), 1741\left(v_{\mathrm{C}=\mathrm{O}}\right.$ cyclic azetidin-2-one $), 2920\left(\mathrm{~V}_{\mathrm{CH}-\mathrm{Cl}}\right),{ }^{1} \mathrm{H}$ NMR: $\delta(\mathrm{ppm}) 1.10\left(3 \mathrm{H}, \mathrm{t}, \mathrm{J}=7.3 \mathrm{HzCH}_{3}\right), 2.80\left(2 \mathrm{H} \mathrm{q}, \mathrm{J}=7.3 \mathrm{~Hz}, \mathrm{CH}_{2}\right), 3.73\left(3 \mathrm{H}, \mathrm{s}, \mathrm{Ar}-\mathrm{O}-\mathrm{CH}_{3}\right), 5.50$ $(1 \mathrm{H}, \mathrm{d}, \mathrm{J}=5.5 \mathrm{~Hz}, \mathrm{~N}-\mathrm{C}-\mathrm{H}$ azetidin-2-one), $5.73(1 \mathrm{H}, \mathrm{d}, \mathrm{J}=5.5 \mathrm{~Hz}, \mathrm{Cl}-\mathrm{CH}$ azetidin-2-one), 6.497.23 (4H, m,Ar-H), ${ }^{13} \mathrm{C}$ NMR: $\delta$ (ppm) 11.96 $\left(\mathrm{CH}_{3}\right.$ acyclic), 27.29 $\left(\mathrm{CH}_{2}\right.$ acyclic $), 111.3-159.2(\mathrm{C}$ of aromatic ring), 56.1 $\left(\mathrm{OCH}_{3}\right), 57.09$ (CHazetidin-2-one), 60.2 (CH-Clazetidin-2-one), 160.4, 157.0 $\left(\mathrm{C}_{2}, \mathrm{C}_{5}\right.$ of thiadiazole ring $), 167.9(\mathrm{C}=\mathrm{O}$ cyclic azetidin-2-one $)$, Anal. Calcd. For: $\mathrm{C}_{14} \mathrm{H}_{14} \mathrm{Cl}$ $\mathrm{N}_{3} \mathrm{O}_{2} \mathrm{~S}: \mathrm{C}, 51.93, \mathrm{H}, 4.36, \mathrm{~N}, 12.98 \%$, found $\mathrm{C}, 51.93, \mathrm{H}, 4.20, \mathrm{~N}, 12.72 \%$, Mass 323.04( $\left(\mathrm{M}^{+}\right)$.

3-Chloro-4-(4-methoxy-phenyl)-1-(5-ethyl-[1,3,4]thiadiazol-2-yl)-azetidin-2-one (2l)

M.P.138-140 ${ }^{\circ}$ C, Yield $65 \%$, IR: $\left(v_{\max } \mathrm{cm}^{-1}\right) 1549\left(v_{\mathrm{C}-\mathrm{C}}\right), 755\left(v_{\mathrm{C}-\mathrm{S}}\right), 1628\left(v_{\mathrm{C}=\mathrm{N}}\right), 1441\left(v_{\mathrm{C}-\mathrm{N}}\right), 3095$ $\left(v_{\mathrm{C}-\mathrm{H}}\right), 1314\left(v_{\mathrm{N}-\mathrm{N}}\right), 1109(v \mathrm{OCH} 3) 1739\left(v_{\mathrm{C}=\mathrm{O}}\right.$ cyclic azetidin-2-one), ${ }^{1} \mathrm{H}$ NMR: $\delta(\mathrm{ppm}) 1.08(3 \mathrm{H}$, $\left.\mathrm{t}, \mathrm{J}=7.3 \mathrm{~Hz}, \mathrm{CH}_{3}\right), 2.80\left(2 \mathrm{H} \mathrm{q}, \mathrm{J}=7.3 \mathrm{~Hz}, \mathrm{CH}_{2}\right), 3.74\left(3 \mathrm{H}, \mathrm{s}, \mathrm{O}-\mathrm{CH}_{3}\right), 5.12(1 \mathrm{H}, \mathrm{d}, \mathrm{J}=5.5 \mathrm{~Hz}, \mathrm{~N}-$ $\mathrm{C}-\mathrm{H}$ azetidin-2-one), $5.50(1 \mathrm{H}, \mathrm{d}, \mathrm{J}=5.5 \mathrm{~Hz}, \mathrm{Cl}-\mathrm{CH}$ azetidin-2-one), 6.89-7.22 (4H, m,Ar-H), ${ }^{13} \mathrm{C}$ NMR $: \delta(\mathrm{ppm}) 12.66\left(\mathrm{CH}_{3}\right.$ acyclic), $27.75\left(\mathrm{CH}_{2}\right.$ acyclic $), 113.7-160.4$ (C of aromatic ring, 55.5 $\left(\mathrm{OCH}_{3}\right), 58.09$ (CHazetidin-2-one), 59.2(CH-Clazetidin-2-one), $161.3, \quad 156.8\left(\mathrm{C}_{2}, \mathrm{C}_{5} \mathrm{Of}\right.$ thiadiazole ring), $167.5\left(\mathrm{C}=\mathrm{O}\right.$ cyclic azetidin-2-one), Anal. Calcd. for: $\mathrm{C}_{14} \mathrm{H}_{14} \mathrm{ClN}_{3} \mathrm{O}_{2} \mathrm{~S}: \mathrm{C}, 51.93$, $\mathrm{H}, 4.36, \mathrm{~N}, 12.98 \%$, found C, 51.80, H, 4.17, N, $12.49 \%$, Mass $323.06\left(\mathrm{M}^{+}\right)$.

\section{Antimicrobial activities}

The synthesized compounds $\mathbf{1}$ and $\mathbf{1 a}-\mathbf{2 l}$ and $\mathbf{2}$ and $\mathbf{2 a}-\mathbf{2 l}$ were evaluated in vitro for antibacterial activity by using filter paper disc diffusion method against different strains of bacteria viz. B. substilis, E. coli and S. tyhpi. All the final product along with standard antibacterial streptomycin were used at 50 and $100 \mathrm{ppm}$ concentrations. Antifungal activity against A.niger, A. Flavus and F. oxisporium at 50 and $100 \mathrm{ppm}$ concentrations by filter paper disc technique. The minimum inhibitory concentration (MIC) values of the synthesized compounds were determined. Standard antibacterial streptomycin and antifungal griseofulvin were also tested under the similar conditions for comparison (Table 2, $3 \& 4$ ). 
Table 2. Antibacterial activity (Inhibition Zone diameter in $\mathrm{mm}$ ) of the compounds $\mathbf{1}$ and 1a-1 $l$ and 2 and $2 a-2 l$

\begin{tabular}{|c|c|c|c|c|c|c|}
\hline \multirow[b]{2}{*}{ Compd. } & \multicolumn{2}{|c|}{ E. coli } & \multicolumn{2}{|c|}{ B. subtilis } & \multicolumn{2}{|c|}{ S. typhi } \\
\hline & $50 \mathrm{ppm}$ & $100 \mathrm{ppm}$ & $50 \mathrm{ppm}$ & $100 \mathrm{ppm}$ & $50 \mathrm{ppm}$ & $100 \mathrm{ppm}$ \\
\hline 1 & 5.0 & 9.0 & 6.0 & 7.5 & 5.0 & 7.0 \\
\hline $1 \mathbf{a}$ & 13.4 & 16.4 & 13.1 & 16.0 & 13.0 & 16.4 \\
\hline 1b & 14.5 & 17.5 & 14.8 & 17.9 & 13.9 & 18.0 \\
\hline 1c & 12.8 & 16.2 & 12.0 & 16.8 & 13.2 & 16.6 \\
\hline 1d & 11.8 & 13.8 & 12.0 & 14.2 & 11.2 & 14.6 \\
\hline $1 e$ & 12.4 & 14.5 & 13.5 & 15.0 & 12.0 & 15.5 \\
\hline 1f & 12.0 & 14.0 & 12.2 & 14.8 & 11.5 & 15.0 \\
\hline $1 \mathrm{~g}$ & 17.2 & 22.2 & 18.0 & 22.0 & 17.0 & 20.8 \\
\hline 1h & 18.5 & 23.5 & 19.0 & 23.8 & 18.0 & 22.5 \\
\hline $1 \mathrm{i}$ & 18.0 & 23.0 & 18.2 & 22.8 & 17.6 & 21.0 \\
\hline $1 \mathbf{j}$ & 10 & 13 & 10 & 12.5 & 9.5 & 13 \\
\hline $1 \mathrm{k}$ & 9.0 & 12 & 10.5 & 13 & 9.0 & 12.5 \\
\hline $1 l$ & 9.5 & 12.5 & 9.5 & 12 & 10 & 13.5 \\
\hline 2 & 6.0 & 9.0 & 7.0 & 9.5 & 7.0 & 9.0 \\
\hline $2 a$ & 14.9 & 17.5 & 14.8 & 16.5 & 14.8 & 17.2 \\
\hline $2 b$ & 15.7 & 18.0 & 15.9 & 17.8 & 15.5 & 18.8 \\
\hline $2 c$ & 14.2 & 17.2 & 14.0 & 16.0 & 14.0 & 17.0 \\
\hline 2d & 12.8 & 15.8 & 11.8 & 15.8 & 12.5 & 14.8 \\
\hline $2 e$ & 13.5 & 16.5 & 12.8 & 16.9 & 13.8 & 15.9 \\
\hline $2 f$ & 13.0 & 16.0 & 12.0 & 16.0 & 13.0 & 15.0 \\
\hline $2 \mathrm{~g}$ & 17.5 & 21.5 & 17.2 & 21.5 & 17.2 & 21.9 \\
\hline $2 \mathrm{~h}$ & 19.0 & 23.5 & 18.5 & 22.5 & 18.5 & 23.2 \\
\hline $2 \mathrm{i}$ & 18.5 & 23 & 18.0 & 22.0 & 18.0 & 22.8 \\
\hline $2 j$ & 10.5 & 13 & 10 & 12.5 & 10 & 13.5 \\
\hline $2 k$ & 10 & 12.5 & 10.5 & 13 & 9 & 12.5 \\
\hline $2 l$ & 9.5 & 12 & 09.5 & 13 & 10 & 14 \\
\hline $\mathbf{S M}^{\mathrm{a}}$ & 24.3 & 26.5 & 21 & 25 & 22 & 26 \\
\hline
\end{tabular}

Table 3. Antifungal activity (Inhibition Zone diameter in $\mathrm{mm}$ ) of the compounds $\mathbf{1}$ and 1a$\mathbf{1} \boldsymbol{l}$ and $\mathbf{2}$ and $\mathbf{2 a - 2 l}$

\begin{tabular}{ccccccc}
\hline Compd. & \multicolumn{2}{c}{ A. flavus } & \multicolumn{2}{c}{ P. citrinum } & \multicolumn{2}{c}{ F. oxysporum } \\
\cline { 2 - 7 } & $50 \mathrm{ppm}$ & $100 \mathrm{ppm}$ & $50 \mathrm{ppm}$ & $100 \mathrm{ppm}$ & $50 \mathrm{ppm}$ & $100 \mathrm{ppm}$ \\
\hline $\mathbf{1}$ & 6.0 & 7.0 & 5.50 & 8.5 & 6.0 & 8.0 \\
$\mathbf{1 a}$ & 13.0 & 16.5 & 14.1 & 17.2 & 13.0 & 16.0 \\
$\mathbf{1 b}$ & 13.5 & 17.0 & 15.0 & 18.0 & 13.3 & 16.8 \\
$\mathbf{1 c}$ & 12.8 & 16.2 & 14.0 & 17.0 & 12.5 & 15.5 \\
$\mathbf{1 d}$ & 12.2 & 14.2 & 10.5 & 13.6 & 11.2 & 14.5 \\
$\mathbf{1 e}$ & 12.6 & 15.5 & 11.8 & 14.8 & 12.2 & 15.8 \\
$\mathbf{1 f}$ & 12.4 & 15.0 & 11.0 & 14.0 & 12.0 & 15.0 \\
$\mathbf{1 g}$ & 16.0 & 20.8 & 16.8 & 20.5 & 18.0 & 21.6 \\
$\mathbf{1 h}$ & 17.0 & 22.5 & 18.0 & 22.2 & 18.5 & 23.5 \\
$\mathbf{1 i}$ & 16.5 & 21.5 & 17.1 & 21.1 & 17.8 & 22.0 \\
$\mathbf{1 j}$ & 10 & 13 & 10 & 13.5 & 10 & 13 \\
$\mathbf{1 k}$ & 9.0 & 12 & 7.0 & 9.5 & 9.0 & 12 \\
\hline
\end{tabular}




\begin{tabular}{|c|c|c|c|c|c|c|}
\hline 11 & 10 & 13.5 & 8.0 & 11 & 10 & 13 \\
\hline 2 & 5.0 & 8.0 & 6.5 & 9.0 & 7.0 & 10 \\
\hline $2 a$ & 13.5 & 17.2 & 14.2 & 17.8 & 14.0 & 17.0 \\
\hline $2 b$ & 14.5 & 18.5 & 15.5 & 18.5 & 14.2 & 17.9 \\
\hline $2 c$ & 13.2 & 18.0 & 14.0 & 17.2 & 13.2 & 16.2 \\
\hline 2d & 12.0 & 13.5 & 11.8 & 14.0 & 12.8 & 15.5 \\
\hline $2 e$ & 12.8 & 14.5 & 12.5 & 14.8 & 13.5 & 16.5 \\
\hline $2 f$ & 12.2 & 14.0 & 12.0 & 14.5 & 13.0 & 16.0 \\
\hline $2 \mathrm{~g}$ & 18.0 & 22.0 & 17.0 & 22.0 & 17.2 & 23.0 \\
\hline $2 \mathrm{~h}$ & 18.8 & 23.5 & 19.0 & 23.8 & 18.0 & 24.0 \\
\hline $2 \mathbf{i}$ & 18.3 & 22.2 & 18.5 & 22.5 & 17.5 & 23.2 \\
\hline $2 \mathrm{j}$ & 10.5 & 13 & 10 & 13 & 11 & 14 \\
\hline $2 \mathrm{k}$ & 9.0 & 12.5 & 7.0 & 10 & 10 & 13 \\
\hline 21 & 11 & 13.5 & 8.5 & 11.5 & 10.5 & 13.5 \\
\hline $\mathbf{G F}^{\mathbf{B}}$ & 24 & 26 & 21 & 25 & 24 & 28 \\
\hline
\end{tabular}

Table 4. Minimal inhibitory concentration $(\mu \mathrm{g} / \mathrm{mL})$ of synthesized compounds against bacterial and fungal strains

\begin{tabular}{ccccccc}
\hline Compd. & E. coli & B. Subtilis & S. Typhi & A. flavus & P.citrinum & F. oxysporum \\
\hline $\mathbf{1}$ & 32.2 & 35.4 & 36.3 & 33.4 & 37.1 & 30.0 \\
$\mathbf{1 a}$ & 16.7 & 17.0 & 16.9 & 13.9 & 15.5 & 15.1 \\
$\mathbf{1 b}$ & 16.1 & 16.4 & 16.2 & 13.1 & 15.3 & 15.0 \\
$\mathbf{1 c}$ & 17.0 & 17.2 & 17.2 & 14.0 & 15.7 & 15.2 \\
$\mathbf{1 d}$ & 19.5 & 20.2 & 21.0 & 16.5 & 18.0 & 18.0 \\
$\mathbf{1 e}$ & 18.2 & 19.5 & 20.3 & 15.5 & 17.0 & 16.7 \\
$\mathbf{1 f}$ & 19.0 & 19.8 & 20.8 & 15.9 & 17.6 & 17.0 \\
$\mathbf{1 g}$ & 15.0 & 16.9 & 15.5 & 12.8 & 14.8 & 13.0 \\
$\mathbf{1 h}$ & 13.5 & 15.6 & 14.1 & 11.3 & 13.2 & 11.9 \\
$\mathbf{1 i}$ & 14.0 & 16.1 & 14.8 & 12.0 & 14.1 & 12.3 \\
$\mathbf{1 j}$ & 27.2 & 27.3 & 27.2 & 22.0 & 25.0 & 23.8 \\
$\mathbf{1 k}$ & 26.5 & 24.2 & 26.3 & 21.5 & 24.3 & 23.1 \\
$\mathbf{1 l}$ & 26.9 & 27.1 & 27.4 & 23.1 & 25.8 & 24.0 \\
$\mathbf{2}$ & 30.0 & 34.2 & 35.0 & 31.1 & 33.5 & 28.8 \\
$\mathbf{2 a}$ & 14.8 & 16.6 & 15.6 & 13.2 & 14.5 & 14.6 \\
$\mathbf{2 b}$ & 14.5 & 16.2 & 15.3 & 13.0 & 14.1 & 14.4 \\
$\mathbf{2 c}$ & 14.9 & 16.2 & 16.0 & 13.9 & 14.8 & 14.9 \\
$\mathbf{2 d}$ & 18.0 & 19.2 & 20.3 & 15.0 & 16.9 & 16.9 \\
$\mathbf{2 e}$ & 17.6 & 18.6 & 19.3 & 14.3 & 16.0 & 16.2 \\
$\mathbf{2 f}$ & 17.9 & 18.9 & 19.8 & 14.5 & 16.3 & 16.8 \\
$\mathbf{2 g}$ & 13.8 & 16.0 & 14.8 & 12.3 & 14.0 & 13.0 \\
$\mathbf{2 h}$ & 12.5 & 15.2 & 14.0 & 11.2 & 13.0 & 12.0 \\
$\mathbf{2 i}$ & 13.2 & 15.3 & 14.3 & 12.0 & 13.8 & 12.7 \\
$\mathbf{2 j}$ & 26.0 & 26.0 & 27.0 & 21.8 & 24.0 & 22.5 \\
$\mathbf{2 k}$ & 25.2 & 25.5 & 26.0 & 20.0 & 23.1 & 21.5 \\
$\mathbf{2 l}$ & 25.8 & 25.8 & 26.2 & 21.2 & 23.8 & 21.8 \\
$\mathbf{S M}$ & 12.0 & 14.0 & 13.0 & - & - & - \\
$\mathbf{G F}$ & - & - & - & 10.0 & 12.0 & 11.0 \\
\hline & & S.M. Straptomycin & G.F.Gresiofulvin & &
\end{tabular}




\section{Results and Discussion}

The compound 2-amino-5-ethyl-1,3,4-thiadiazoleon reaction with selected several substituted aromatic aldehydes produced benzylidene-(5-ethyl-[1,3,4]thiadiazol-2-yl)-amine compounds 1 and $\mathbf{1 a}-1 \boldsymbol{l}$. The characteristic absorption for Schiff base in IR spectra of compounds 1 and 1a-1l appeared in the range of $1635-1670 \mathrm{~cm}^{-1}$ and in the ${ }^{1} \mathrm{H}$ and ${ }^{13} \mathrm{C}$ NMR spectra, signal appeared at $\delta$ 7.52-8.31 and $\delta$ 158.5.-160.4 ppm N=C acyclic Schiff base and 156.7,160.7 ppm (thiadiazole) respectively.In the ${ }^{1} \mathrm{H}$ NMR spectrum of compound $\mathbf{1}$ a broad signal of $\mathrm{NH}_{2}$ has been disappeared. The compounds $\mathbf{1}$ and $\mathbf{1 a}-1 \boldsymbol{l}$ on treatment with $\mathrm{ClCH}_{2} \mathrm{COCl}$ in the presence of $\mathrm{Et}_{3} \mathrm{~N}$ furnished final products compounds $\mathbf{2}$ and $\mathbf{2 a}-\mathbf{2 l}$ In the IR spectra of compounds $\mathbf{2}$ and $\mathbf{2 a}-\mathbf{2 l}$ carbonyl group of $\boldsymbol{\beta}$-lactam ring showed characteristic absorption in the range of $1735-1745 \mathrm{~cm}^{-1}$ and ${ }^{1} \mathrm{H}$ NMR spectra of compounds 2 and $2 \mathbf{a}-2 l$ showed two doublet for $(\mathrm{N}-\mathrm{CH})$ and $(\mathrm{CH}-\mathrm{Cl})$ in the range $\delta$ 5.085.50, 5.50-5.94 ppm, respectively. In ${ }^{13} \mathrm{C}$ NMR spectra of compounds 2 and $2 \mathbf{a}-2 \boldsymbol{l}$ three characteristic signals appeared for $(\mathrm{N}-\mathrm{CH}),(\mathrm{CH}-\mathrm{Cl})$ and $(\mathrm{CO}$ cyclic) in the range of $\delta 56.2-$ 58.6, 58.8-60.2 and 167.5-169.8 ppm, respectively. The IR absorption, ${ }^{1} \mathrm{H}$ and ${ }^{13} \mathrm{C}$ NMR signals of $\mathrm{N}=\mathrm{CH}$ have been disappeared. The compounds $\mathbf{2}$ and $\mathbf{2 a - 2} \boldsymbol{l}$ shows stereoisomerism, spectral data as well as literature support the synthesis of diastereomer of azetidine in good yield. These all fact collectively indicates for the synthesis of all above compounds. Spectral and physical data of compounds $\mathbf{1 a}-1 \boldsymbol{l}$ and $\mathbf{2}$ and $\mathbf{2 a}-\mathbf{2} \boldsymbol{l}$ are given as supplementary data(Table-1). All the synthesised compounds were also confirmed by their mass spectral analysis. The results of the all described activities (antibacterial and antifungal) were summarized in Tables 2 and 3. The results of the antimicrobial screening data revealed that all the compound $\mathbf{1 a}-1 \boldsymbol{l}$ and $\mathbf{2}$ and $\mathbf{2 a}-\mathbf{2} \boldsymbol{l}$ showed considerable and varied activity against the selected microorganism. A 3-Chloro-1-(5-ethyl-[1,3,4]thiadiazol-2-yl)-4phenyl-azetidin-2-one, compound $\mathbf{2}$ and $\mathbf{2 a}-2 \boldsymbol{l}$ were prepared and screened for their antimicrobial activity data (as shown in tables 2 and 3) revealed that all the synthesized compound $\mathbf{1 a}-\mathbf{1} \boldsymbol{l}$ and $\mathbf{2}$ and $\mathbf{2 a}-2 \boldsymbol{l}$ have a structure activity relationship (SAR) because activity of compounds varies with substitution. Nitro group containing compounds $(\mathbf{2} \mathbf{h}>\mathbf{2} \mathbf{i}$ $>\mathbf{2 g}$ ) showed higher activity than chloro( $\mathbf{2 b}>\mathbf{2 a}>\mathbf{2 c}$ ), or bromo group containing compounds (2e $>\mathbf{2 f}>\mathbf{2 d}$ ). Similar order of activity in compound 1a-1l. Chloro and bromo derivatives also have higher activity than other rested compounds and we also observed in antibacterial and antifungal activity table 2 and 3 anti microbial activity of compound $\mathbf{2}$ and $\mathbf{2 a}-\mathbf{2} \boldsymbol{l}$ higher then compound $\mathbf{1}$ and $\mathbf{1 a - 1} \mathbf{l}$. On the basis of SAR, concluded that the activity of compounds depends on electron withdrawing nature of the substituted groups.

\section{Conclusions}

At the conclusion, a new series of compound $\mathbf{2}$ and $\mathbf{2 a - 2 1}$ were successfully synthesized and all the synthesized final compounds screened for their spectral and in-vitro biological study. The investigation of antimicrobial (antibacterial and antifungal) activities data revealed that the compounds $(\mathbf{2} \mathbf{h}>\mathbf{2 i}>\mathbf{2 g}),(\mathbf{2 b}>\mathbf{2 a}>\mathbf{2 c})$ and (2e) displayed excellent activity, the compounds $(\mathbf{2} \mathbf{f}>\mathbf{2} \mathbf{d})$ showed moderate activity and rested compounds showed less activity compared with standard drugs.

\section{Acknowledgement}

The authors are thankful to SAIF, Punjab University, Chandigarh (India) for providing spectral and analytical data of the compounds. We are also grateful to Mr. Vahid-Ul-Hassan, research scholar department of Zoology Dr. H.S. Gour Central University, Sagar [M.P.] for 
providing help in carrying out the antimicrobial screening. We are also thankful to Head, Department of Chemistry, Dr. H. S. Gour Central University Sagar (India) for giving the facilities to carry out the work and UGC-New Delhi (India), for financial assistance as R.G.N.F. fellowship.

\section{References}

1. Dua R, Shrivastava S, Sonwane S K and Srivastava S K, Adv Biol Res., 2011, 5(3), 120-144.

2. Malleshappa N, Noolvi Harun M, Patel Sarita Kamboj and Swaranjit Singh Cameotra, Arabian J Chem., 2016, 9(11), S1283-S1289; DOI:10.1016/j.arabjc.2012.02.003

3. Karakuş S and Rollas S, Farmaco II, 2002, 57(6), 577-581; DOI:10.1016/S0014827X(02)01252-1

4. Al-Qahtani, Yunus M Siddiqui, Adnan A Bekhit, Ola A El-Sayed, Hassan Y AboulEnein and Mohammed N Al-Ahdal, Saudi Pharm J., 2009, 17(3), 227-232; DOI:10.1016/j.jsps.2009.08.005

5. Maddila S, Gorle S, Sampath Ch and Lavanya P, J Saudi Chem Soc., 2016, 20, S306S312; DOI:10.1016/j.jscs.2012.11.007

6. Silvia Schenone, Chiara Brullo, Olga Bruno, Francesco Bondavalli, Angelo Ranise, Walter Filippelli, Barbara rinaldi, Annalisa Capuano and Giuseppe Falcone, Bioorg Med Chem., 2006,14(6), 1698-1705; DOI:10.1016/j.bmc.2005.10.064

7. Yosuf M, khan R A and Ahmed B, Bioorg Med Chem., 2008, 16(17), 8029-8034; DOI:10.1016/j.bmc.2008.07.056

8. Zhao J, Chen B O, Shi Y P, Liu Y M, Zhao H C and J Cheng J, Chin Chem Lett., 2012, 23(7), 817-819; DOI:10.1016/j.cclet.2012.04.005

9. Katarina Jakovljevic, Ivana Z Matic, Tatjana Stanojkovic, Ana Krivokuc, Violeta Markovic, Milan D Joksovic, Nevena Mihailovic, Marija Nic'iforovic and Ljubinka Joksovic, Bioorg Med Chem Lett., 2017, 27(16), 3709-3715;

DOI:10.1016/j.bmcl.2017.07.003

10. Kikkeri P Harish, Kikkeri N Mohana and Lingappa Mallesha, Drug Invention Today, 2013, 5(2), 92-99; DOI:10.1016/j.dit.2013.06.002

11. Navin B Patel and Jaymin C Patel, Ara J Chem., 2011, 4(4), 403-411; DOI:10.1016/j.arabjc.2010.07.005

12. Anand K Halve, Deepti Bhadaura and Rakesh Dudey, Bioorg Med Chem Lett., 2007, 17(2), 341-345; DOI:10.1016/j.bmcl.2006.10.064

13. Karthikeyan Elumalai, Mohammed Ashraf Ali, Manogaran Elumalai, Kalpana Eluri, Sivaneswari Srinivasan, Sujit Kumar Mohanti and Anil Thota, Drug Invention Today, 2013, 5(2), 100-104; DOI:10.1016/j.dit.2013.05.007

14. Srivastava S K, Srivatsava S and Srivastava S D, Indian J Chem., 1999, 38B, 183-187.

15. Kumar A, Gurtu S, Agrawal J C, Sinha J N, Bhargava K P and Shankar K, J Indian Chem Soc, 1983, 60, 608-609.

16. Jaishree V, Ramdas N, Sachin J and Ramesh B, J Saudi Chem Soc., 2012, 16(4), 371376; DOI:10.1016/j.jscs.2011.02.007

17. Mounyr Balouirin, Moulay Sadiki and Saad Koraichi Ibnsouda, J Pharm Anal., 2016, 6(2), 71-79; DOI:10.1016/j.jpha.2015.11.005

18. Omer Erturk, Biologia, Bratislava, Section Cell Mol Biol., 2006, 61(3), 275-278; DOI:10.2478/s11756-006-0050-8 